\title{
ON INFINITE-DIMENSIONAL LINEAR SPACES ${ }^{(1)}$
}

\author{
BX \\ GEORGE W. MACKEY
}

\section{INTRODUCTION}

Let $X$ be an abstract linear space and let $X^{*}$ be the space of all linear functionals defined on $X$. Associated with each norm defined on $X$ is its "norm set," the subspace $L$ of $X^{*}$ consisting of those linear functionals which are continuous with respect to it. Our starting point is the observation that two norms in $X$ define the same topology if and only if their norm sets are identical. This observation suggests two lines of investigation which turn out to be rather closely related. First of all it makes it possible to regard a normable topological linear space as a linear space together with a distinguished family of linear functionals rather than as a linear space with a topology, and suggests investigating such spaces from this point of view. In the second place it makes it appear desirable to find out just what subspaces of $X^{*}$ are norm sets. Now this latter problem involves a study of the subspaces of a linear space, and as will be shown below the problem of classifying the subspaces of an abstract linear space is quite trivial. However $X^{*}$ is not an abstract linear space but has added structure by virtue of being a space of linear functionals. Furthermore, and this is what is of interest here, this structure may be regarded as being induced in $X^{*}$ by distinguishing a subspace of $X^{* *}$. In precisely what sense this is true will appear below. Thus both lines of investigation lead to a consideration of the object obtained from an abstract linear space by distinguishing a subspace of its conjugate. Such an object we call a linear system. It is the purpose of this paper to take the first steps toward the development of a systematic theory of linear systems. Although the structure induced in an abstract linear space by converting it into a linear system can almost always be obtained by introducing a suitable (not usually normable) topology instead, so that linear systems are not strictly speaking new mathematical objects, the notion does lead to a rather unconventional point of view. We turn now to a discussion of a feature of this point of view which plays an important role in our entire development.

Let $X$ and $X^{*}$ be as above. For each member $x$ of $X$ the function $f$ on $X^{*}$

Presented to the Society, April 28, 1944; received by the editors March 30, 1944.

(1) Most of the results in this paper have been drawn from the author's doctoral thesis, Harvard, 1942. Some of the work on the latter was done while the author was a Sheldon travelling fellow from Harvard University in residence at the California Institute of Technology and the Institute for Advanced Study. A general description of the contents of the present paper will be found in [17]. Numbers in brackets refer to the Bibliography at the end of the paper. 
such that $f(l)=l(x)$ for all $l$ in $X^{*}$ is clearly a member of $X^{* *}$. Thus $X$ may be regarded as a subspace of $X^{* *}$. Let $\left(X^{*}\right)_{X}$ denote the linear system obtained from $X^{*}$ by distinguishing the members of $X^{* *}$ contained in $X$. Finally for each subspace $L$ of $X^{*}$ let $X_{L}$ denote the linear system into which it converts $X$. Then if the notions of isomorphism and automorphism are defined for linear systems in the obvious fashion it is easy to show that the linear systems $X_{L}$ and $X_{M}$ are isomorphic if and only if there is an automorphism of the linear system $\left(X^{*}\right)_{X}$ under which the map of $L$ is $M$. Thus the problem of classifying the linear systems definable on a fixed linear space is identical with the problem of classifying the subspaces of a certain linear system, and methods occurring naturally in one may be applied to the other. In particular every definition which we may make differentiating between linear systems carries with it a definition distinguishing between subspaces of linear systems of the form $\left(X^{*}\right)_{X}$ and conversely. We shall make tacit use of this phenomenon throughout this paper in that we shall in general make only one of each pair of dual definitions explicitly. For instance we shall define a "boundedly closed" subspace of an $X^{*}$ in a certain way and then without further ado speak of boundedly closed linear systems. Furthermore we shall often find it convenient to use both of the equivalent statements " $X_{L}$ is boundedly closed" and " $L$ is boundedly closed" in the course of the same discussion without explicitly reminding the reader that they mean exactly the same thing.

The problem of classifying completely the subspaces of any but the most trivial linear systems and the problem of classifying completely all linear systems, including as they do the corresponding problems for normed linear spaces, appear to be hopelessly difficult. In this paper we shall be concerned rather with the less ambitious project of singling out certain broad classes of linear systems and subspaces of linear systems which seem to us to be important and of finding out what we can about the relationships existing, first between the various classes as classes, and second between the individual members of each class, paying particular attention to questions of the following form: if a linear system (subspace of a linear system) has a certain property and a certain operation is performed upon the system (subspace) does the result continue to have this property? Some of the results obtained have applications to the theory of normed linear spaces and these are indicated where they occur. There are more extensive applications to the theory of convex topological linear spaces. These will be presented in another paper a summary of which has already been published [18].

In greater detail the contents of the present paper are as follows. Chapter I contains some introductory matter on abstract linear spaces and linear functionals. In Chapter II the notion of linear system is introduced and some elementary definitions and theorems are presented. Most of the general theory of linear systems is developed in Chapters III through VI. The two principal notions involved are those of closed subspace and bounded subset. In Chap- 
ter III various theorems about closed subspaces which are needed in subsequent chapters are proved and a detailed discussion of the modularity relation in the lattice of all closed subspaces of a linear system is given. Chapters IV, $\mathrm{V}$, and VI concern themselves with various notions arising out of the notion of bounded set. It turns out that different linear systems defined on the same linear space may have the same bounded subsets. Chapter IV is concerned with the relationship of these to a certain maximal one called the bounded closure of the others and also with a certain very closely related convergence notion. In Chapter V linear systems are classified according to the natures of their families of bounded subsets and the various kinds of linear systems so introduced are studied in some detail. Chapter VI is concerned with a notion of uniform boundedness obtained by comparing the boundedness in a linear system with that in its "conjugate system." In the seventh and last chapter the results of the earlier chapters are applied to obtain information about norm sets. The paper is concluded with a list of unsolved problems.

The problems which we treat do not seem to have been studied to any great extent by other authors. Boas and Tukey in [4] and Fichtenholz in [6] have considered the question "When is an $L$ in an $X^{*}$ a norm set?" and have obtained some results distinct from ours and in rather a different spirit. Also in an as yet unpublished work on topological linear spaces N. Bourbaki in introducing what he calls the "weak duality" between such spaces uses a notion essentially equivalent to our linear system which, however, he does not study in any detail. Finally in [1] Arnold explicitly introduces the notion of a linear space with a distinguished family of linear functionals but confines himself to a study of the ring of all "bounded" linear operators on such a space. On the other hand we are indebted to a large majority of the writers on linear spaces for the ideas we have obtained from reading their treatments of other problems. In this connection we should like to mention particularly a paper of G. Sirvint [22] which had considerable influence on our Chapter VI.

\section{Chapter I. Preliminaries}

1. Abstract linear spaces and their subspaces. By a linear space we shall always mean a real linear space, and by a subspace of a linear space a subset which is closed under the two linear space operations. A subset of a linear space whose elements are linearly independent and which is properly contained in no other subset with this property we shall call a Hamel basis. It is an immediate consequence of a form of a lemma of Zorn $\left(^{2}\right)$ that every linear space admits a Hamel basis $\left({ }^{3}\right)$. It has been shown by Löwig [15] that any two

(2) See [24, p. 7].

(3) This is stated but not proved by Banach [2, p. 231] and others. However the proof given by Hamel [7] for the case of the real number system regarded as a linear space over the rationals generalizes at once. 
Hamel bases for the same linear space have the same cardinal number. This cardinal numer we shall call the dimension $\left(^{4}\right)$ of the space. It is clear that if $\left\{\phi_{\alpha}\right\}$ is a Hamel basis for a linear space $X$ then every element $x$ of $X$ has a unique representation of the form: $x=c_{\alpha_{1}} \phi_{\alpha_{1}}+c_{\alpha_{2}} \phi_{\alpha_{2}}+\cdots+c_{\alpha_{n}} \phi_{\alpha_{n}}$ where each $c_{\alpha_{i}}$ is a real number. Thus if $X$ has dimension $\aleph$ and $S$ is any set of elements of cardinal $\aleph$ then $X$ is isomorphic in the sense of abstract algebra to the linear space $\mathcal{F}$ of all real valued functions on $S$ which vanish on all but a finite number of points of $S$. It follows at once that two linear spaces are isomorphic if and only if they have the same dimension. Since the space $\mathcal{F}$ may be defined for any set $S$ and obviously has the cardinal of $S$ for its dimension it is clear that there exists a linear space of every dimension. In other words there is a natural one-to-one correspondence between abstract linear spaces and cardinal numbers. Inasmuch as practically all of the commonly studied topological linear spaces, linear lattices, and so on have the same dimension, namely $C$, it is clear that they may be regarded as having been obtained from a single abstract linear space by introducing different topologies, different partial orderings, and so on.

Let $M$ be a subspace of the linear space $X$. The method used to prove the existence of a Hamel basis may be used equally well to show that any Hamel basis for $M$ is contained in one for $X$. It follows at once that there exists a subspace $N$ of $X$ such that $M \cap N=0$ and( $\left.{ }^{5}\right) M+N=X$. We call $N$ a complement of $M$. It is clear that $N$ is unique only if $M=0$ or $M=X$ but since $N$ is obviously isomorphic to the quotient space $X / M$ it follows that all complements of $M$ have the same dimension. We call this dimension the deficiency of $M$. It is clear that the sum of the dimension and the deficiency of $M$ is the dimension of $X$ and that given any two cardinal numbers $\boldsymbol{\aleph}_{1}$ and $\boldsymbol{N}_{2}$ whose sum is the dimension of $X$ there exists a subspace of $X$ whose dimension and deficiency are $\boldsymbol{\aleph}_{1}$ and $\boldsymbol{\aleph}_{2}$ respectively. Finally it is clear that a subspace is completely characterized by its dimension and deficiency in the sense that if $M$ and $N$ are subspaces with the same dimension and the same deficiency then there is an automorphism of $X$ under which the map of $M$ is $N$.

2. Linear functionals. By a linear functional $\left({ }^{6}\right)$ on a linear space $X$ we shall always mean a real valued function $l$ defined throughout $X$ such that $l(\lambda x+\mu y)=\lambda l(x)+\mu l(y)$ whenever $x$ and $y$ are in $X$ and $\lambda$ and $\mu$ are real numbers. If $\left\{\phi_{\alpha}\right\}$ is any Hamel basis for $X$ and $f$ is any real valued function defined on $\left\{\phi_{\alpha}\right\}$ it is obvious that there is a unique linear functional $l$ on $X$ such that $l\left(\phi_{\alpha}\right)=f\left(\phi_{\alpha}\right)$ for all $\alpha$ and hence that linear functionals on $X$ exist

(4) This is what Löwig calls the affine dimension.

(5) By $M+N$ we mean the smallest subspace containing $M$ and $N$. Moreover if $A$ is an arbitrary subset of a linear space then by $A+$ we mean the smallest subspace containing $A$. We call $A+$ the linear span of $A$.

(6) Our use of the word linear differs from that of many writers in that it has no topological implications. 
in great abundance. Furthermore if addition and multiplication by reals are defined for linear functionals in the obvious fashion it is readily verified that the family of all linear functionals on $X$ is itself a linear space. We shall call this space the conjugate of $X$ and denote it by $X^{*}$. If $l$ is a member of $X^{*}$ it is obvious that the set of all $x$ in $X$ such that $l(x)=0$ is a subspace. We shall call it the null space of $l$. If $l$ is not identically zero it is readily verified that its null space has deficiency one.

3. The dimensions of some particular linear spaces. Löwig [15] has shown that whenever $X$ is a linear space with more than $C$ elements then its dimension is equal to the number of its elements. If we use this theorem or the following, the dimensions of all the common linear spaces may be computed without difficulty.

THEOREM I-1. If $X$ is a nonfinite-dimensional complete normed linear space then the dimension of $X$ is at least $C$.

Proof. Let $l_{1}$ be a nonzero continuous linear functional defined on $X$ and let $M_{1}$ be its null space. Let $x_{1}$ be any element of $X-M_{1}$ such that $\left\|x_{1}\right\|=1$. Let $l_{2}$ be a nonzero continuous linear functional defined on $M_{1}$ and let $M_{2}$ be the null space of $l_{2}$. Let $x_{2}$ be any element of $M_{1}-M_{2}$ such that $\left\|x_{2}\right\|=1 / 2$. Continuing this process by induction it is clear that one may define a sequence of elements of $X, x_{1}, x_{2}, \cdots$, such that for each $n=1,2, \cdots,\left\|x_{n}\right\|=1 / 2^{n}$ and $x_{n+1}, x_{n+2}, \cdots$ are all contained in a closed subspace of $X$ which excludes $x_{1}, x_{2}, \cdots, x_{n}$. Let $\lambda_{1}, \lambda_{2}, \cdots$ be an arbitrary bounded sequence of real numbers. It is clear that $\lambda_{1} x_{1}, \lambda_{1} x_{1}+\lambda_{2} x_{2}, \lambda_{1} x_{1}+\lambda_{2} x_{2}+\lambda_{3} x_{3}, \cdots$ is a convergent sequence of members of $X$ and accordingly, since $X$ is complete, has a limit in $X$. The mapping from the linear space of bounded sequences into $X$ defined in this manner obviously preserves linear combinations. Furthermore if $\lambda_{1} x_{1}+\lambda_{2} x_{2}+\cdots=0$ for any bounded sequence $\lambda_{1}, \lambda_{2}, \cdots$ then $\lambda_{1} x_{1}=-\lambda_{2} x_{2}-\lambda_{3} x_{3}-\cdots$ and since $x_{2}, x_{3}, \cdots$ are contained in a closed subspace excluding $x_{1}, \lambda_{1}$ must be zero. By the same argument $\lambda_{2}$ must be zero and continuation by induction leads to a proof that $\lambda_{1}, \lambda_{2}, \ldots$ is the null sequence. Hence the mapping is one-to-one and is accordingly an isomorphism. Thus the dimension of $X$ is at least as great as that of the space of bounded sequences. But the sequences $t, t^{2}, t^{3}, \ldots$ where $0<t<1$ are clearly linearly independent. Hence the dimension of $X$ is at least $C$.

The reader may now readily verify the following statements. The dimension of a separable complete normed linear space is either finite or $C$. If $S$ is a set of cardinal $\aleph, \mathcal{S}$ is the linear space of all real valued functions on $S$, and $B$ is the linear space of all bounded real valued functions on $S$, then $S$ and $B$ are isomorphic and their common dimension is $\aleph$ or 2 according as $\aleph$ is finite or infinite. If $X$ is a linear space of dimension $\aleph$ then the dimension of $X^{*}$ is $\aleph$ or $2^{\aleph}$ according as $\aleph$ is finite or infinite. 


\section{Chapter II. Linear systems}

1. Fundamental definitions. By a linear system we shall mean a linear space together with a distinguished subspace of its conjugate space. If $X$ is a linear space and $L$ is a subspace of $X^{*}$ the symbol $X_{L}$ will denote the linear system defined on $X$ by distinguishing $L$. If $X$ and $Y$ are two linear spaces and $T$ is a linear transformation from $X$ into $Y$ then for each linear functional $f$ in $Y^{*}$ the function $l$ defined on $X$ such that $l(x)=f(T(x))$ for all $x$ in $X$ is a member of $X^{*}$ which we shall denote by $T^{*}(f)$. If $L$ and $M$ are subspaces of $X^{*}$ and $Y^{*}$ respectively then $T$ will be said to be a homomorphism of the linear system $X_{L}$ into the linear system $Y_{M}$ if $T^{*}(M) \subseteq L$. If in addition $T(X)=Y$, $T^{*}(M)=L$, and $T$ is one-to-one, $T$ will be said to be an isomorphism. If an isomorphism exists $X_{L}$ and $Y_{M}$ will be said to be isomorphic. It is clear that the relationship of isomorphism is reflexive symmetric and transitive. An isomorphism of a linear system with itself will be called an automorphism.

If $X_{L}$ is a linear system and $Y$ is a subspace of $X$ then every member of $X^{*}$ has associated with it the member of $Y^{*}$ which coincides with it throughout $Y$. The linear system $Y_{L}$ obtained from $Y$ by distinguishing the members of $Y^{*}$ associated with members of $L$ in this fashion will be called a subsystem of $X_{L}$. Similarly every member of $X^{*}$ which vanishes throughout $Y$ has associated with it a member of $(X / Y)^{*}$. The linear system $X_{L} / Y$ obtained from $X / Y$ by distinguishing the members of $(X / Y)^{*}$ associated with members of $L$ in this manner will be called the quotient system of $X_{L} \bmod Y$. It is clear that the natural mapping of $X$ onto $X / Y$ is a homomorphism of $X_{L}$ onto the quotient system of $X_{L} \bmod Y$. Let $X_{L}$ and $Y_{M}$ be two linear systems. Let $Z$ be the set of all pairs $x, y$ with $x \in X$ and $y \in Y$ converted into a linear space in the obvious fashion. For each pair $l, m$ where $l \in L$ and $m \in M$ there is a unique member $f$ of $Z^{*}$ such that $f(x, 0)=l(x)$ for all $x$ in $X$ and $f(0, y)=m(y)$ for all $y$ in $Y$. The set of all such linear functionals is a subspace $N$ of $Z^{*}$. We shall call the linear system $Z_{N}$ the direct union of $X_{L}$ and $Y_{M}$ and denote it by $X_{L} \oplus Y_{M}$.

If $X_{L}$ is a linear system and $x \in X$ the function $f_{x}$ such that $f_{x}(l)=l(x)$ for all $l$ in $L$ is easily seen to be a member of $L^{*}$ and the set of all such members to be a subspace. The linear system obtained from $L$ by distinguishing these members of $L^{*}$ will be called the conjugate of $X_{L}$ and will be denoted by the symbol $X_{L}^{\circ}$, or if no confusion can arise sometimes by the symbol $L_{X}$. The mapping $x \rightarrow f_{x}$ is a linear mapping of $X$ onto the linear space underlying $X_{L}^{\circ}$. It is readily verified that this mapping is a homomorphism of $X_{L}$ onto $X_{L}^{\circ \circ}$ and that if in particular $f_{x}=0$ implies that $x=0$ then it is an isomorphism. Now $f_{x}=0$ implies $x=0$ if and only if $l(x)=0$ for all $l$ in $L$ implies $x=0$; that is, if and only if $L$ is what Banach calls a total subspace of $X^{*}$ [2, p. 42]. Whenever $L$ is total we shall say that $X_{L}$ is a regular linear system. Since it is obvious that the conjugate of any linear system is regular it follows 
from the above that $X_{L}^{\circ \circ}$ and $X_{L}$ are isomorphic if and only if $X_{L}$ is regular. In any case we shall refer to $X_{L}^{\circ \circ}$ as the regularization of $X_{L}$. It is clear that any linear system is isomorphic to the direct union of its regularization with a linear system with only the zero functional distinguished. We shall not always maintain the logical distinction between $X_{L}$ and $X_{L}^{\circ \circ}$ but shall often regard members of $X$ as identical with the functionals which they define. In this sense, when $X_{L}$ is regular, $X_{L}$ and $X_{L}^{\circ \circ}$ are identical and $X_{L}$ and $X_{L}^{\circ}$ are mutually conjugate linear systems.

2. Automorphisms and isomorphisms. In this section we state three elementary theorems about automorphisms and isomorphisms of linear systems. The proofs of two of these depend upon the following lemma whose truth is an immediate consequence of the definitions concerned. The proof of the third depends upon some notions to be developed in the next chapter and will be deferred.

Lemma. Let $X_{L}$ and $Y_{M}$ be regular linear systems, let $T$ be a homomorphism of $X_{L}$ into $Y_{M}$, and let $T^{*}$ be the function on $M$ to $L$ introduced in defining homomorphism. Then $T^{*}$ is a homomorphism of $Y_{M}^{\circ}$ into $X_{L}^{\circ}$ and $T$ is an isomorphism if and only if $T^{*}$ is an isomorphism.

THEOREM II-1. If $X_{L}$ and $Y_{M}$ are regular linear systems then $X_{L}$ and $Y_{M}$ are isomorphic if and only if $X_{L}^{\circ}$ and $Y_{M}^{\circ}$ are isomorphic.

Proof. If $T$ is an isomorphism between $X_{L}$ and $Y_{M}$ then by the lemma $T^{*}$ is an isomorphism between $X_{L}^{\circ}$ and $Y_{M}^{\circ}$. If $X_{L}^{\circ}$ and $Y_{M}^{\circ}$ are isomorphic then by the first part of the theorem so are $X_{L}^{\circ \circ}$ and $Y_{M}^{\circ \circ}$. But $X_{L}^{\circ \circ}$ and $Y_{M}^{\circ \circ}$ are isomorphic to $X_{L}$ and $Y_{M}$ respectively.

TheOREM II-2. If $X$ is a linear space and $L$ and $M$ are subspaces of $X^{*}$ then $X_{L}$ and $X_{M}$ are isomorphic linear systems if and only if there exists an automorphism of the linear system $\left(X^{*}\right)_{X}$ under which the map of $M$ is $L$.

Proof. Let $T$ be an isomorphism between $X_{L}$ and $X_{M} . T$ is then an automorphism of the linear space $X$ and clearly also of the linear system $X_{X}$. By the lemma $T^{*}$ is then an automorphism of $\left(X^{*}\right)_{X}$. By the definition of isomorphism $T^{*}(M)=L$. Conversely suppose that there exists an automorphism $T$ of $\left(X^{*}\right)_{X}$ such that $T(M)=L . T^{*}$ is then an automorphism of $X$ which is clearly an isomorphism between $X_{L}$ and $X_{M}$.

THEOREM II-3. Let $X_{L}$ be a regular linear system and let $x_{1}, x_{2}, \cdots, x_{n}$; $y_{1}, y_{2}, \cdots, y_{n}$ be two sets of $n$ linearly independent elements of $X, n=1,2, \cdots$. Then there exists an automorphism $T$ of $X_{L}$ such that $T\left(x_{i}\right)=y_{i}$ for each $i=1$, $2, \cdots, n$.

In particular Theorem II-3 says that the group of automorphisms of a regular linear system is transitive on the nonzero elements. This fact applied 
to the linear system $\left(X^{*}\right)_{X}$ has the interesting consequence that the only linear systems which may be defined on an abstract linear space by an expression of the form " $L$ consists of all members of $X^{*}$ which have such and such a property" are $X_{X}$. and $X_{0}$ (where 0 is the zero-dimensional subspace of $X^{*}$ ). To define other linear systems on such a space one must somewhere use an expression like "let such and such be an arbitrary such and such."

3. The linear system of a normed linear space. Let $X$ be a linear space. Each norm in $X$. defines a topology with respect to which the members of a certain subspace $L$ of $X^{*}$ become continuous. We shall call $X_{L}$ the linear system associated with the corresponding normed linear space or simply the linear system of the normed linear space. The following slight generalization of a theorem due to Fichtenholz [6] enables us to establish the connection between normed linear spaces and their linear systems referred to in the introduction.

TheOREM II-4. If $X$ and $Y$ are normed linear spaces, $X_{L}$ and $Y_{M}$ are their linear systems, and $T$ is a linear transformation from $X$ into $Y$, then $T$ is continuous if and only if $T$ is a homomorphism from $X_{L}$ into $Y_{M}$.

Proof. If $T$ is continuous and $m$ is any member of $M$ it is clear that $m(T(x))$ is a continuous function of $x$. In other words $T^{*}(m)$ is in $L$ and $T$ is a homomorphism. Conversely suppose that $T$ is a homomorphism and that $\left\|x_{n}\right\| \rightarrow 0$ where $x_{n} \in X$ for $n=1,2, \ldots$. Let $\left\{\gamma_{n}\right\}, n=1,2, \ldots$, be a sequence of positive real numbers such that $\gamma_{n} \rightarrow 0$ and $\left\{\left\|x_{n} / \gamma_{n}\right\|\right\}$ is bounded. Let $m$ be an arbitrary member of $M$ and let $T^{*}(m)=l$. Then $\left\{\left|m\left(T\left(x_{n} / \gamma_{n}\right)\right)\right|\right\}$ $=\left\{\left|l\left(x_{n} / \gamma_{n}\right)\right|\right\}$ and is bounded since $l$ is in $L$. Hence by a well known theo$\operatorname{rem}\left({ }^{7}\right)$ on normed linear spaces $\left\{\left\|T\left(x_{n} / \gamma_{n}\right)\right\|\right\}$ is bounded. It follows at once that $\left\|T\left(x_{n}\right)\right\| \rightarrow 0$ and hence that $T$ is continuous.

When $X$ and $Y$ are built on the same abstract linear space and $T$ is the identity, this theorem reduces to that of Fichtenholz. It follows in particular that two norms in the same space $X$ give the same topology if and only if the same members of $X^{*}$ are continuous with respect to both and the following is an immediate corollary.

THEOREM II-5. If $X$ and $Y$ are normed linear spaces and $X_{L}$ and $Y_{M}$ are their linear systems then $X$ and $Y$ are isomorphic as normed linear spaces if and only if $X_{L}$ and $Y_{M}$ are isomorphic linear systems.

On the basis of Theorem II-5 and well known properties of normed linear spaces the following may be established. The proof is left to the reader.

Theorem II-6. If $X$ and $Y$ are normed linear spaces and $X_{L}$ and $Y_{M}$ are

(7) Theorème 6 on page 80 of [2]. In proving this theorem only the completeness of the conjugate space is used and as is well known the conjugate of any normed linear space is complete. 
their linear systems then the linear system of the direct product of $X$ and $Y$ is isomorphic to $X_{L} \oplus Y_{M}$. If $Z$ is a subspace of $X$ then the linear system of $Z$ as a normed linear space is isomorphic to the linear system $Z_{L}$ and if $Z$ is closed the linear system of the quotient space $X / Z$ is isomorphic to the linear system $X_{L} / Z$.

On the other hand it is not in general true that the linear system of the conjugate of a normed linear space is isomorphic to the conjugate of the linear system of the space. As a matter of fact these two linear systems are isomorphic if and only if the normed linear space is reflexive. We observe also that every normed linear space, reflexive or not, has a regular linear system.

\section{Chapter III. Closed linear subspaces}

1. The operation ' and the closure of a subspace. Let $X_{L}$ be a linear system. If $M$ denotes a subspace of $X(L), M^{\prime}$ will be used to denote the set of all $l$ in $L(x$ in $X)$ such that $l(x)=0$ for all $x(l)$ in $M$. It is clear that $M^{\prime}$ is always a subspace, that $M^{\prime \prime} \supseteq M$ and that whenever $M_{1}$ and $M_{2}$ are subspaces with $M_{1} \subseteq M_{2}$ then $M_{1}^{\prime} \supseteq M_{2}^{\prime}$. Thus $M^{\prime \prime \prime}=\left(M^{\prime}\right)^{\prime \prime} \supseteq M^{\prime}$ and since $M^{\prime \prime} \supseteq M$, $\left(M^{\prime \prime}\right)^{\prime}=M^{\prime \prime \prime} \subseteq M^{\prime}$. In other words $M^{\prime \prime \prime}=M^{\prime}$ and we conclude $\left.{ }^{8}\right)$ that $M=M^{\prime \prime}$ if and only if $M$ is of the form $N^{\prime}$. If $M$ is a subspace of $X, M^{\prime \prime}$ will be called the closure of $M$. It is easy to see that if $M$ is a subspace of $L$. then $M^{\prime \prime}$ is the closure of $M$ relative to the linear system $X_{L}^{\circ}$. If $M=M^{\prime \prime}$, $M$ will be said to be closed. It is clear that in any case $M^{\prime \prime}$ is closed and is contained in every closed subspace which contains $M$; that is, is the smallest closed subspace which contains $M$.

THEOREM III-1. If $\left\{M_{\alpha}\right\}$ is a family of closed subspaces of a linear system $X_{L}$ then $\prod M_{\alpha}$ is also closed. If $M$ is a closed subspace of $X_{L}$ and $N$ is a finitedimensional subspace of $X$ then $M+N$ is closed.

Proof. Since $\prod M_{\alpha} \subseteq M_{\alpha}$, $\left(\prod M_{\alpha}\right)^{\prime \prime} \subseteq M_{\alpha}^{\prime \prime}=M_{\alpha}$. Hence $\left(\prod M_{\alpha}\right)^{\prime \prime} \subseteq \prod M_{\alpha}$ and so $\left(\prod M_{\alpha}\right)^{\prime \prime}=\prod M_{\alpha}$. To prove the second statement it is clearly sufficient to show that if $M$ is closed and $\bar{x}$ is not in $M$ then $M \dot{+} \bar{x}$ is closed. Since $\bar{x}$ is not in $M^{\prime \prime}$, there exists $\bar{l}$ in $M^{\prime}$ with $\bar{l}(\bar{x})=1$. For each $l$ in $M^{\prime}$, $l(\bar{x})-\bar{l}(\bar{x}) l(\bar{x})=l(\bar{x})-l(\bar{x})=0$ so that $l-l(\bar{x}) \bar{l}$ is in $(M \dot{+} \bar{x})^{\prime}$. Hence for each $y$ in $(M+\bar{x})^{\prime \prime}, l(y)-l(\bar{x}) \bar{l}(y)=0$. In other words if $y$ is in $(M \dot{+} \bar{x})^{\prime \prime}$ then $l(y-\bar{l}(y) \bar{x})=0$ for all $l$ in $M^{\prime}$ so that $y-\bar{l}(y) \bar{x}$ is in $M^{\prime \prime}=M$ and $y$ is in $M \dot{+} \bar{x}$.

Since the closure of the 0 -dimensional subspace of $X$ is $L^{\prime}$ this theorem has the following corollary.

COROLLARY. All the finite-dimensional subspaces of $X_{L}$ are closed if and only if $X_{L}$ is regular.

2. The lattice of a linear system. It follows from the remarks of the pre-

(8) Cf. [3, p. 24] for an abstract discussion of this sort of argument. 
ceding section that the closed subspaces of a linear system $X_{L}$ form a complete lattice under partial ordering by inclusion. We call this the lattice of the system. It follows also that the operation ' sets up a one-to-one inclusion inverting correspondence between the closed subspaces of $X_{L}$ and the closed subspaces of $X_{L}^{\circ}$. In other words it sets up an anti-isomorphism [3, p. 8] between the lattice of $X_{L}$ and that of $X_{L}^{\circ}$.

If $\mathcal{L}$ is any lattice which has a largest element $I$ and a smallest element 0 we shall call a system of elements $x_{1}, x_{2}, \cdots, x_{n-1}$ such that $x_{1}>x_{2}>\cdots>x_{n-1}>0$ a chain of length $n$ joining $x_{1}$ to 0 and a system such that $x_{1}<x_{2}<\cdots<x_{n-1}<I$ a chain of length $n$ joining $x_{1}$ to $I$. It follows from Theorem III-1 that if $M$ is an element of the lattice of a linear system then the chains joining $M$ to $I$ (to 0 ) are bounded in length if and only if $M$ has finite deficiency (is finite-dimensional) and moreover that the upper bound, if it exists, is the deficiency (dimension) of $M$. Since an anti-automorphism associates chains to $I$ with chains to 0 and vice versa the truth of the following theorem is now apparent.

THEOREM III-2. If $X_{L}$ is a linear system and $n$ is a positive integer then for every n-dimensional closed subspace of $X_{L}, M^{\prime}$ has deficiency $n$ in $L$ and for every closed subspace of $X_{L}$ of deficiency $n, M^{\prime}$ is $n$-dimensional.

3. Characterization of a linear system by its lattice. It is a corollary of Theorem III-2 that a subspace of a linear space has deficiency one if and only if it is the null space of a nonzero linear functional, that this linear functional is unique up to a numerical factor, and that if we are dealing with a linear system the subspace is closed if and only if the linear functionals are distinguished. Thus the distinguished linear functionals are determined as soon as the closed subspaces of deficiency one are known. In particular, two linear systems constructed on the same linear space are identical if and only if they have the same closed subspaces. Accordingly a linear system may be alternatively regarded as an abstract linear space certain of the subspaces of which have been designated as closed. As the reader may readily verify a family $\mathcal{F}$ of subspaces of a linear space $X$ is the family of closed subspaces relative to some linear system constructed on $X$ if and only if it has the following properties: (a) If $\left\{M_{\alpha}\right\}$ is a set of members of $\mathcal{f}$ then $\prod M_{\alpha}$ is a member of $\mathcal{F}$. (b) If $M$ is in $\mathcal{F}$ and $x$ is in $X$ then $M+x$ is in $\mathcal{F}$. (c) Every $M$ in $\mathcal{F}$ is an intersection of members of $\mathcal{F}$ which have deficiency one.

Actually the closed subspaces of a linear system determine the system in a much stronger sense, as the following theorem shows.

Theorem III-3. If $X_{L}$ and $Y_{M}$ are two linear systems and $\mathcal{L}_{1}$ and $\mathcal{L}_{2}$ are their respective lattices then $X_{L}$ and $Y_{M}$ have isomorphic regularizations if and only if $\mathcal{L}_{1}$ and $\mathcal{L}_{2}$ are isomorphic as abstract lattices. 
Proof. If $X_{L}$ and $Y_{M}$ have isomorphic regularizations they have isomorphic conjugates. Hence the duals of their respective lattices are isomorphic and hence these lattices are isomorphic. Thus we have proved half of the theorem and in the other half may suppose that $X_{L}$ and $Y_{M}$ are regular. Let there be an isomorphism between $\mathcal{L}_{1}$ and $\mathcal{L}_{2}$. It follows from the remarks concerning chains made in the preceding section that this isomorphism sets up a one-to-one dimension preserving correspondence between the finitedimensional subspaces of $X$ and $Y$ respectively. Hence it sets up a one-to-one linear independence preserving correspondence between their one-dimensional subspaces. Now if $X$ and $Y$ are finite-dimensional the theorem is an obvious consequence of the fact that all finite-dimensional subspaces of a regular linear system are closed. Hence we may suppose that $X$ and $Y$ are not less than three-dimensional and, applying Lemma $A$ of [16], deduce the existence of a linear transformation $T$ from all of $X$ onto all of $Y$ which gives the same correspondence between one-dimensional subspaces as that set up by the lattice isomorphism. It is clear that if $X_{1}$ is any closed subspace of $X_{L}$ then $T\left(X_{1}\right)$ is the closed subspace of $Y_{M}$ which corresponds to $X$ under the lattice isomorphism. Thus in particular $T$ sets up a one-to-one correspondence between the closed subspaces of $X_{L}$ of deficiency one and the closed subspaces of $Y_{M}$ of deficiency one. Hence by the remarks at the beginning of this section $T$ must set up an isomorphism between $X_{L}$ and $Y_{M}$.

As will appear below, a subspace of a normed linear space is topologically closed if and only if it is a closed subspace of the linear system of the space. Thus the theorem of [16] to the effect that a normed linear space is determined to within isomorphism by its lattice of closed subspaces is a corollary of Theorems II-5 and III-3. The other two theorems of [16] have similar generalizations to linear systems, the group theorem in particular taking on a slightly simpler form. As we shall not need to make use of them and as their proofs are somewhat involved and do not differ essentially from those of their specializations to normed linear spaces we shall confine ourselves to their statements.

TheOREM( $\left.{ }^{9}\right)$ III-4. Let $X_{L}$ and $Y_{M}$ be regular linear systems. Let $R_{1}$ be the ring of all homomorphisms of $X_{L}$ into itself and let $R_{2}$ be the corresponding ring for $Y_{M}$. Then $X_{L}$ and $Y_{M}$ are isomorphic linear systems if and only if $R_{1}$ and $R_{2}$ are isomorphic as abstract rings.

TheOREM III-5. Let $X_{L}$ and $Y_{M}$ be regular linear systems. Let $G_{1}$ be the group of automorphisms of $X_{L}$ and let $G_{2}$ be that of $Y_{M}$. Then $G_{1}$ and $G_{2}$ are isomorphic as abstract groups if and only if $X_{L}$ is isomorphic either to $Y_{M}$ or to $Y_{M}^{\circ}$.

4. Modularity in the lattice of a linear system. Let $\mathcal{L}$ be a lattice. $\mathcal{L}$ is

(9) The corresponding theorem for complete normed linear spaces is due to Eidelheit [5]. 
said to be modular if whenever $a \leqq c$ the following equality holds: ${ }^{*}(a \cup b) \cap c$ $=a \cup(b \cap c)$. In any case Wilcox [28] calls the ordered pair $b, c$ modular if * holds for all $a$ with $a \leqq c$. Dualizing Wilcox's definition we shall call the ordered pair $a, b d$-modular if * holds for all $c$ with $a \leqq c$. It is clear that an ordered pair of elements is modular ( $d$-modular) if and only if the corresponding pair in the dual of $\mathcal{L}$ is $d$-modular (modular) and that the following statements about $\mathcal{L}$ are equivalent: (1) $\mathcal{L}$ is modular. (2) Every ordered pair in $\mathcal{L}$ is modular. (3) Every ordered pair in $\mathcal{L}$ is $d$-modular. On the other hand, as will appear below, it is not in general true that modularity and $d$-modularity are equivalent.

TheOREM III-6. Let $X_{L}$ be a linear system and let $\mathcal{L}$ be its lattice. If $M$ and $N$ are members of $\mathcal{L}$ then $M, N$ is a d-modular pair if and only if $M+N$ is closed.

Proof. Suppose that $M+N$ is closed and that $K$ is any member of $\mathcal{L}$ with $M \subseteq K$. Let $x$ be any member of $X$ with $x \in(M \cup N) \cap K$. Then since $x \in M \cup N=M+N, x=m+n$ where $m \in M$ and $n \in N$. But $x \in K$ and $m \in M \subseteq K$. Hence $n \in K$. Hence $n \in K \cap N$. Thus $x \in M+(N \cap K) \subseteq M$ $\cup(N \cap K)$. In other words $(M \cup N) \cap K \subseteq M \cup(N \cap K)$ and since the reverse inequality holds in any lattice $M, N$ is a $d$-modular pair. Conversely suppose that $M, N$ is a $d$-modular pair and that $M+N$ is not closed. Let $\bar{x}$ be an element of $X$ in $M \cup N$ and not in $M \dot{+} N$. Then $K=M \dot{+} \bar{x}$ is in $\mathcal{L}$ and properly contains $M$. Hence $(M \cup N) \cap K=M \cup(N \cap K)$. Now since $\bar{x} \in M \cup N$ the left-hand side reduces to $K$. On the other hand if $x \in N \cap K$ then $x=m+\lambda \bar{x}$ where $m \in M$ and $x \in N$. Hence $\lambda \bar{x}=x-m \in M+N$ so that $\lambda=0$. In other words $x \in M$. Thus the right-hand side reduces to $M$ and since $K$ properly contains $M$ this is a contradiction.

COROLlaRY 1. The lattice of a linear system is modular if and only if whenever $M$ and $N$ are closed subspaces of the system then $M+N$ is also closed.

CoRollary 2. If $X_{L}$ is a linear system such that $M+N$ is a closed subspace whenever $M$ and $N$ are, then the conjugate system also has this property.

Proof. Corollary 2 follows from Corollary 1 and the fact from lattice theory that the dual of a modular lattice is modular.

CoRollary 3. If $X$ is a linear space and $M$ and $N$ are closed subspaces of the linear system $\left(X^{*}\right)_{X}$ then $M+N$ is also closed.

COROLLARY 4. In the lattice of closed subspaces of a linear system the relation of d-modularity is symmetric.

The relation of modularity itself may also be interpreted for the lattice of a linear system but not quite so readily. It is first necessary to introduce some further notions. Let $T$ be a homomorphism of the linear system $X_{L}$ into a linear system $Y_{M}$. Let $N$ be the null space of $T$ and let $H$ be the natural 
mapping of $X$ onto the quotient space $X / N$. Finally let $T^{\prime}$ be the unique linear transformation fron $X / N$ onto $Y$ such that $T^{\prime}(H(x))=T(x)$ for all $x$ in $X$. Then $T^{\prime}$ is one-to-one from $X / N$ onto the range $R$ of $T$ and, as is easily verified, is a homomorphism of the quotient system of $X_{L} \bmod N$ onto the subsystem $R$ of $Y_{M}$. Following the terminology used in the theory of topological groups we shall call $T$ an open homomorphism whenever $T^{\prime}$ is an isomorphism between these two linear systems. Now let $M$ and $N$ be two closed subspaces of $X_{L}$. It is readily verified that the mapping $m, n \rightarrow m+n$ of the linear system $M_{L} \oplus N_{L}$ onto the linear system $(M \dot{+} N)_{L}$ is a homomorphism which, as will appear below, may not be open. Whenever it is not open we shall say that $M$ and $N$ are tangent. The property of being not tangent is the important one. The following lemma enables us to describe it directly in terms of the relationship between $M, N$ and $L$.

LeMma. $M$ and $N$ are not tangent if and only if whenever $l_{1}$ and $l_{2}$ are members of $L$ such that $l_{1}(x)=l_{2}(x)$ for all $x$ in $M \cap N$ then there exists an $l$ in $L$ such that $l(x)=l_{1}(x)$ for all $x$ in $M$ and $l(x)=l_{2}(x)$ for all $x$ in $N$.

Proof. Let $H$ designate the homomorphism of $M_{L} \oplus N_{L}$ onto $(M+N)_{L}$ described above. It follows at once from the definitions concerned that $H$ is open if and only if for every distinguished linear functional $f$ on $M_{L} \oplus N_{L}$ which vanishes on the null space of $H$ there exists $l$ in $L$ such that $f(x)$ $=l(H(x))$ for all $x$ in $M_{L} \oplus N_{L}$. Now the null space of $H$ is the set of all pairs $z,-z$ with $z \in M \cap N$ and $f$ is distinguished if and only if there exists $l_{1}$ and $l_{2}$ in $L$ such that $f(m, n)=l_{1}(m)+l_{2}(n)$ for all $m$ in $M$ and $n$ in $N$. Such an $f$ vanishes on $z,-z$ if and only if $l_{1}(z)=l_{2}(z)$. Thus $H$ is open if and only if whenever $l_{1}$ and $l_{2}$ are members of $L$ such that $l_{1}(z)=l_{2}(z)$ for all $z$ in $M \cap N$ then there exists $l$ in $L$ such that $l(m+n)=l_{1}(m)+l_{2}(n)$ whenever $m \in M$ and $n \in N$ or, what amounts to the same thing, $l(m)=l_{1}(m)$ for all $m$ in $M$ and $l(n)=l_{2}(n)$ for all $n$ in $N$. But this is what was to be proved.

We are now in a position to give the promised interpretation of modularity.

TheOREM III-7. Let $X_{L}$ be a linear system and let $\mathcal{L}$ be its lattice. If $M$ and $N$ are members of $\mathcal{L}$ then $M, N$ is a modular pair if and only if $M$ and $N$ are not tangent.

Proof. Suppose that $M$ and $N$ are not tangent and that $l_{1} \in L$ vanishes on $M \cap N$. Applying the lemma to $l_{1}$ and 0 we conclude that there exists $l$ in $L$ such that $l(x)=l_{1}(x)$ for all $x$ in $M$ and $l(x)=0$ for all $x$ in $N$. Writing $l_{1}=\left(l_{1}-l\right)+l$ we see that $l_{1} \in M^{\prime}+N^{\prime}$. Suppose conversely that whenever $l(M \cap N)=0$ then $l \in M^{\prime}+N^{\prime}$. Let $l_{1}$ and $l_{2}$ be members of $L$ such that $l_{1}(x)=l_{2}(x)$ : for all $x$ in $M \cap N$. Then $l_{1}-l_{2}=l_{3}+l_{4}$ where $l_{3} \in M^{\prime}$ and $l_{4} \in N^{\prime}$. Hence $l_{1}-l_{3}=l_{2}+l_{4}$. Let $l=l_{1}-l_{3}=l_{2}+l_{4}$. Then $l(x)=l_{1}(x)$ for all $x$ in $M$ and 
$l(x)=l_{2}(x)$ for all $x$ in $N$. In other words the condition of the lemma is satisfied and $M$ and $N$ are not tangent. Thus $M$ and $N$ are not tangent if and only if $(M \cap N)^{\prime}=M^{\prime}+N^{\prime}$. Now by elementary lattice theory $(M \cap N)^{\prime}=M^{\prime} \cup N^{\prime}$. Furthermore $M^{\prime} \cup N^{\prime}=M^{\prime \prime}+N^{\prime}$ if and only if $M^{\prime}+N^{\prime}$ is closed; that is, if and only if $M^{\prime}, N^{\prime}$ is a $d$-modular pair. But as we have already pointed out $M^{\prime}$ and $N^{\prime}$ are $d$-modular if and only if $M$ and $N$ are modular. This completes the proof of the theorem.

COROLLARY 1. The lattice of a linear system is modular if and only if there are no two closed subspaces of the system which are tangent.

COROLlaRY 2. In the lattice of closed subspaces of a linear system the relation of modularity is symmetric.

Although modularity and $d$-modularity are not equivalent $\left({ }^{10}\right)$, even in the lattice of a linear system, there are two slightly stronger notions which are equivalent in such a lattice. Let $J, K, M$ and $N$ be closed subspaces of a linear system $X_{L}$ such that $J \subseteq M \subseteq K$ and $M / J$ and $K / M$ are one-dimensional. Then if $M+N$ is closed, $K+N=(M+x)+N=(M+N)+x$ is closed by virtue of Theorem III-1. In other words, whenever $M, N$ is a $d$-modular pair so is $K, N$. Similarly whenever $M, N$ is a modular pair so is $J, N$. In fact if $M, N$ is modular then $M^{\prime}, N^{\prime}$ is $d$-modular and $J^{\prime} / M^{\prime}$ is one-dimensional. Hence by the above argument $J^{\prime}, N^{\prime}$ is $d$-modular so that $J, N$ must be modular. On the other hand it is not in general true that the $d$-modularity of $J, N$ follows from that of $M, N$ or that the modularity of $K, N$ follows from that of $M, N$. Accordingly we make the following definitions. If $M, N$ is a modular pair of closed subspaces and for each closed subspace $K$ such that $M \subseteq K$ and $K / M$ is one-dimensional $K, N$ is also a modular pair, we shall say that $M, N$ is a stable modular pair. If $M, N$ is a $d$-modular pair of closed subspaces and for each closed subspace $J$ such that $J \subseteq M$ and $M / J$ is one-dimensional $J, N$ is also a $d$-modular pair, we shall say that $M, N$ is a stable $d$-modular pair. It is clear that $M, N$ is a stable modular ( $d$-modular) pair if and only if $M^{\prime}, N^{\prime}$ is a stable $d$-modular (modular) pair.

THeOREM III-8. Let $X_{L}$ be a linear system and let $M$ and $N$ be members of the lattice $\mathcal{L}$ of $X_{L}$. Then the following statements about $M$ and $N$ are equivalent. (1) $M, N$ is a stable modular pair. (2) $M, N$ is a stable d-modular pair. (3) $M, N$ is both a modular pair and a d-modular pair.

Proof. (a) (3) implies (1). Suppose that (3) holds and that $K$ is any member of $\mathcal{L}$ such that $M \subseteq K$ and $K / M$ is one-dimensional. We shall show that $K, N$ is modular, specifically that $(K \cap N)^{\prime} \subseteq K^{\prime}+N^{\prime}$ and hence that $M, N$ is a stable modular pair. Let $l$ be any member of $(K \cap N)^{\prime}$. Then $l$ is a member

(10) This together with our other negative statements about modularity will be verified by examples in $\$ 6$ of this chapter. 
of $(M \cap N)^{\prime}$ and hence, since $M, N$ is modular, of $M^{\prime}+N^{\prime}$. Thus $l=l_{1}+l_{2}$ where $l_{1} \in M^{\prime}$ and $l_{2} \in N^{\prime}$. If $K \subseteq M+N$ then $K=M+\bar{x}$ where $\bar{x} \in N \cap K$. Thus $l(\bar{x})=0$ and $l_{2}(\bar{x})=0$ so that $l_{1}(\bar{x})=0$. In other words $l_{1} \in K^{\prime}$ so that $l \in K^{\prime} \dot{+} N^{\prime}$. If on the other hand $K \cap(M+N) \neq K$ then $K=M \dot{+} \bar{x}$ where $\bar{x} \notin M+N$. Since $M, N$ is a $d$-modular pair, $M \dot{+} N$ is closed and there exists $l_{3}$ in $L$ such that $l_{3}(M \dot{+} N)=0$ and $l_{3}(\bar{x})=l_{1}(\bar{x})$ and hence such that $l_{1}-l_{3} \in K^{\prime}$ and $l_{2}+l_{3} \in N^{\prime}$. Since $l=l_{1}+l_{2}=\left(l_{1}-l_{3}\right)+\left(l_{2}+l_{3}\right)$ it follows that $l \in K^{\prime}+N^{\prime}$. Thus in any case $(K \cap N)^{\prime} \subseteq K^{\prime}+N^{\prime}$ and this is what was to be proved.

(b) (1) implies (3). Suppose that (1) holds. Let $\bar{x}$ be any member of $M \cup N$ which is not in $M$. Then since $M \subseteq M+\overline{+} \bar{x}$ and $M \dot{+} \bar{x}, N$ is modular we have $(M \cup N) \cap(M \dot{+} \bar{x})=M \cup(N \cap(M \dot{+} \bar{x}))$ or, since $M \dot{+} \bar{x} \subseteq M \cup N$, $M \dot{+} \bar{x}=M \cup(N \cap(M \dot{+} \bar{x}))$. It follows then that $M \cup(N \cap(M \dot{+} \bar{x})) \neq M$ and hence that there exists $m$ in $M$ so that $m+\bar{x} \in N$. Thus $\bar{x} \in M+N$. In other words $M \cup N \subseteq M+N$ so that $M+N$ is closed and $M, N$ is $d$-modular as weli as modular.

(c) (2) and (3) are equivalent. $M, N$ satisfies (3) if and only if $M^{\prime}, N^{\prime}$ satisfies (3). By (a) and (b), $M^{\prime}, N^{\prime}$ satisfies (3) if and only if $M^{\prime}, N^{\prime}$ satisfies (1). But $M^{\prime}, N^{\prime}$ satisfies (1) if and only if $M, N$ satisfies (2). Thus (2) and (3) are equivalent and the proof of the theorem is complete.

CoRollary. $M, N$ is a stable modular (d-modular) pair if and only if whenever $M_{1}$ and $N_{1}$ are "near" to $M$ and $N$ in the sense that $M /\left(M \cap M_{1}\right)$, $M_{1} /\left(M \cap M_{1}\right), N /\left(N \cap N_{1}\right)$, and $N_{1} /\left(N \cap N_{1}\right)$ are all finite-dimensional then $M_{1}, N_{1}$ is a modular (d-modular) pair.

The notion of nontangency or modularity for pairs of closed subspaces is particularly interesting in the case in which they are disjoint( $\left.{ }^{11}\right)$. For reasons which will become clear in $\S 6$ we say that two closed subspaces which are disjoint and nontangent are completely disjoint. It follows easily from the theory developed above that if $M$ and $N$ are closed subspaces of the linear system $X_{L}$ then $M$ and $N$ are nontangent if and only if the maps of $M$ and $N$ under the natural homomorphism of $X_{L}$ on $X_{L} /(M \cap N)$ are completely disjoint. Thus the notion of complete disjointness may be used instead of that of nontangency in interpreting modularity.

The following theorem which will be of use in what follows is an easy consequence of the definitions concerned. We leave the proof to the reader.

Theorem III-9. Let $M$ and $N$ be disjoint closed subspaces of ihe regular linear system $X_{L}$. Then the following statements about $M$ and $N$ are equivalent: (1) $M$ and $N$ are completely disjoint. (2) If $T_{1}$ is an arbitrary homomorphism of $M_{L}$ into itself and $T_{2}$ is an arbitrary homomorphism of $N_{L}$ into itself then the

(11) We say that two subspaces of a linear space are disjoint if they are as near to being disjoint in the set theoretic sense as possible; that is, if their intersection is the zero element. It is clear that disjoint closed subspaces can exist only in regular linear systems. 
unique linear transformation $T$ of $M+N$ into itself which coincides on $M$ with $T_{1}$ and on $N$ with $T_{2}$ is a homomorphism of $(M+N)_{L}$ into itself. (3) There exist distinct real numbers $\lambda_{1}$ and $\lambda_{2}$ such that the unique linear transformation $T$ of $M+N$ into itself which is $\lambda_{1}$ times the identity in $M$ and $\lambda_{2}$ times the identity in $N$ is a homomorphism of $(M \dot{+} N)_{L}$ into itself.

5. Further theorems on automorphisms. We are now in a position to prove Theorem II-3 and to present some theorems about automorphisms whose statements depend upon the notion of closure. We begin by doing the former.

Proof of Theorem II-3. Let $M=x_{1}+x_{2}+\cdots+x_{n}+y_{1}+y_{2}+\cdots+y_{n}$. By Theorem III-2, $M^{\prime}$ has finite deficiency in $L$. Let $N$ be any complement of $M^{\prime}$. Then $N$ is finite-dimensional and hence closed. Since $M^{\prime \prime}+N=L$ $=M^{\prime} \cup N, M^{\prime \prime} \cap N^{\prime}=L^{\prime}=0$ and since $M^{\prime} \cap N=0, M^{\prime \prime} \cup N^{\prime}=X$. But since $M$ is finite-dimensional $M^{\prime \prime}=M$ and $M^{\prime} \dot{+} M$ is closed. Hence $M \cap N^{\prime}=0$ and $M \dot{+} N^{\prime}=X$. Since $M^{\prime} \dot{+} N=M^{\prime} \cup N, M$ and $N^{\prime}$ are modular and hence completely disjoint. Thus if $T_{1}$ is any automorphism of $M_{L}$ and $T$ is the unique linear transformation on $X$ coinciding on $M$ with $T_{1}$ and on $N^{\prime}$ with the identity it follows from Theorem III-9 that $T$ is an automorphism of $X_{L}$. Accordingly it is sufficient to prove the theorem for the finite-dimensional linear system $M_{L}$. But in a finite-dimensional regular linear system, since all subspaces are closed, all linear functionals are distinguished. Thus every automorphism of $M$ as a linear space is also an automorphism of $M_{L}$. Since the truth of the theorem for linear space automorphisms is well known the proof is now complete.

TheOREM III-10. Let $X_{L}$ be a regular linear system and let $n$ be a positive integer. Then if $M$ and $N$ are both n-dimensional subspaces of $X_{L}$ or are both closed subspaces of $X_{L}$ of deficiency $n$ there exists an automorphism $T$ of $X_{L}$ such that $T(M)=N$.

Proof. When $M$ and $N$ are both finite-dimensional the theorem is an immediate corollary of Theorem II-3. When $M$ and $N$ are closed and have deficiency $n$ then, by Theorem III-2, $M^{\prime}$ and $N^{\prime}$ are both $n$-dimensional. Hence by the first part of this theorem there exists an automorphism $T_{1}$ of $X_{L}^{\circ}$ such that $T_{1}\left(N^{\prime}\right)=M^{\prime}$. It follows from the lemma preceding Theorem II-1 that $T=T_{1}^{*}$ is an automorphism of $X_{L}$. Furthermore each of the following statements is clearly equivalent to its predecessor: $x$ is in $M . l(x)=0$ for all $l$ in $M^{\prime}$. $T_{1}(l)(x)=0$ for all $l$ in $N^{\prime} . l(T(x))=0$ for all $l$ in $N^{\prime} . T(x)$ is in $N^{\prime \prime}=N$. Thus $T(M)=N$ and the proof is complete.

CoRollary 1. If $X_{L}$ is a regular linear system, $n$ is a positive integer, and $M$ and $N$ are closed subsystems having deficiency $n$ then $M_{L}$ and $N_{L}$ are isomorphic linear systems. 
Corollary 2. Let $X_{L}$ be a regular linear system. Then the following statements about $X_{L}$ are equivalent. (a) There exists a closed subspace $M$ of $X_{L}$ of deficiency one such that $M_{L}$ and $X_{L}$ are isomorphic linear systems. (b) $X_{L}$ is isomorphic to its direct union with a one-dimensional regular linear system. (c) If $M$ is any closed subspace of $X_{L}$ with finite deficiency then $M_{L}$ and $X_{L}$ are isomorphic linear systems. (d) $X_{L}$ is isomorphic to its direct union with any finite-dimensional regular linear system.

We shall call a linear system with any one and hence all of the above properties a stable linear system.

LEMMA. Let $X_{L}$ be a regular linear system such that both $X$ and $L$ have dimension $\aleph_{0}$. Then there exist Hamel bases $\left\{x_{n}\right\}$ and $\left\{l_{n}\right\}, n=1,2, \cdots$, for $X$ and $L$ respectively such that $l_{i}\left(x_{j}\right)=\delta_{i}^{j}$ for $i, j=1,2, \cdots$.

Proof $\left({ }^{12}\right)$. Let $\left\{y_{n}\right\}$ and $\left\{m_{n}\right\}, n=1,2, \cdots$, be arbitrary Hamel bases for $X$ and $L$ respectively. We define $\left\{x_{n}\right\}$ and $\left\{l_{n}\right\}$ by induction. Let $l_{1}=m_{1}$ and let $x_{1}=y_{n_{1}} / m_{1}\left(y_{n_{1}}\right)$ where $n_{1}$ is the first integer such that $m_{1}\left(y_{n_{1}}\right) \neq 0$. Suppose that $x_{1}, x_{2}, \cdots, x_{k}$ and $y_{1}, y_{2}, \cdots, y_{k}$ have been defined. If $k$ is odd let $n_{0}$ be the first integer such that $y_{n_{0}} \notin x_{1}+x_{2}+\cdots+x_{k}$ and let $x_{k+1}=y_{n_{0}}-\left(l_{k}\left(y_{n_{0}}\right) x_{k}+\cdots+l_{1}\left(y_{n_{0}}\right) x_{1}\right)$. Then let $\bar{n}$ be the first integer such that $m_{\tilde{n}}\left(x_{k+1}\right) \neq 0$ and let $l_{k+1}=\left(m_{\bar{n}}-\left(m_{\bar{n}}\left(x_{k}\right) l_{k}+\cdots+m_{n}\left(x_{1}\right) l_{1}\right)\right) / m_{n}\left(x_{k+1}\right)$. If $k$ is even let $n_{0}$ be the first integer such that $m_{n_{0}} \notin l_{1}+l_{2}+\cdots+l_{k}$ and let $l_{k+1}=m_{n_{0}}-\left(m_{n_{0}}\left(x_{k}\right) l_{k}+\cdots+m_{n_{0}}\left(x_{1}\right) l_{1}\right)$. Then let $\bar{n}$ be the first integer such that $l_{k+1}\left(y_{\tilde{n}}\right) \neq 0$ and let $x_{k+1}=\left(y_{\tilde{n}}-\left(l_{k}\left(y_{\tilde{n}}\right) x_{k}+\cdots+l\left(y_{\tilde{n}}\right) x_{1}\right)\right) / l_{k+1}\left(y_{\tilde{n}}\right)$. It follows at once by induction that $l_{i}\left(x_{j}\right)=\delta_{i}^{j}$ for $i, j=1,2, \cdots$, and in particular that the $x_{i}$ and $l_{j}$ are linearly independent. Finally it is clear that $X=x_{1}+x_{2} \dot{ }+\cdots$ and $L=l_{1}+l_{2} \dot{ }+\cdots$. Thus $\left\{x_{n}\right\}$ and $\left\{l_{n}\right\}$ are Hamel bases with the desired property and the proof of the lemma is complete.

Theorem III-11. Let $X$ be an $\boldsymbol{\aleph}_{0}$-dimensional linear space and let $M$ and $N$ be $\aleph_{0}$-dimensional subspaces of $X^{*}$. Then there exists an automorphism $T$ of the linear system $\left(X^{*}\right)_{X}$ such that $T(M)=N$ if and only if $M^{\prime}$ and $N^{\prime}$ have the same dimension. Furthermore given any subspace $K$ of $X$ whose deficiency is $\aleph_{0}$ there exists an $\aleph_{0}$-dimensional subspace $L$ of $X^{*}$ such that $L^{\prime}=K$.

Proof. If $T$ exists then, by Theorem II-2, $X_{M}$ and $X_{N}$ are isomorphic linear systems. Hence $M^{\prime}$ and $N^{\prime}$ must have the same dimension. Suppose conversely that $M^{\prime}$ and $N^{\prime}$ have the same dimension, say $\alpha$ where $\alpha=1,2, \cdots, \aleph_{0}$. Let $J$ be a complement of $M^{\prime}$ in $X$. Then it is clear that $X_{M}$ is isomorphic to the direct union of $J_{M}$ with an $\alpha$-dimensional linear system with no nonzero distinguished linear functionals. Similarly $X_{N}$ is isomorphic to the direct union of $K_{N}$ with an $\alpha$-dimensional linear system with no nonzero distinguished linear functionals where $K$ is a complement of $N^{\prime}$. Now $K_{N}$ and $J_{M}$

(12) Cf. [10, p. 293]. 
are regular linear systems and $K, J, N$, and $M$ are all $\boldsymbol{\aleph}_{0}$-dimensional. Hence it follows at once from the above lemma that $K_{N}$ and $J_{M}$ are isomorphic linear systems. Thus $X_{N}$ and $X_{M}$ are isomorphic linear systems so that by Theorem II-2 a $T$ with the required properties exists. Finally let $K$ be any subspace of $X$ whose deficiency is $\boldsymbol{N}_{0}$ and let $J$ be a complement. Let $x_{1}, x_{2}, \ldots$ be a Hamel basis for $J$ and for each $n=1,2, \ldots$ let $l_{n}$ be the unique linear functional on $X$ such that $l_{n}\left(x_{j}\right)=\delta_{n}^{j}$ for $j=1,2, \cdots$ and $l_{n}(x)=0$ for all $x$ in $K$. Then $L=l_{1}+l_{2}+\cdots$ is $\aleph_{0}$-dimensional and $L^{\prime}=K$.

Corollary. If $X_{L}$ and $Y_{M}$ are regular linear systems such that $X, Y, L$, and $M$ are $\aleph_{0}$-dimensional then $X_{L}$ and $Y_{M}$ are isomorphic.

We leave the proof of the following extension of Theorem III-11 to the reader.

Theorem III-12. Let $X$ be an $\boldsymbol{\aleph}_{0}$-dimensional linear space, let $M$ and $N$

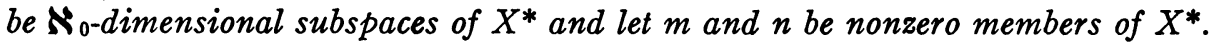
Then there exists an automorphism $T$ of $\left(X^{*}\right)_{X}$ such that $T(M)=N$ and $T(m)=n$ if and only if $M^{\prime}$ and $N^{\prime}$ have the same dimension and one of the following conditions holds. (a) $m \in M$ and $n \in N$. (b) $m \in M^{\prime \prime}-M$ and $n \in N^{\prime \prime}-N$. (c) $m \notin M^{\prime \prime}$ and $n \notin N^{\prime \prime}$.

6. Connections with the theory of normed linear spaces. Let $X$ be a normed linear space and let $X_{L}$ be its linear system. It follows at once from the Hahn-Banach extension theorem [2, p. 55] that a subspace $M$ of $X$ is topologically closed if and only if it is an intersection of null spaces of members of $L$; that is, if and only if it is closed as a subspace of $X_{L}$. Since there may be continuous $\left({ }^{13}\right)$ linear functionals on $L$ not representable by members of $X$ the closed( $\left.{ }^{13}\right)$ subspaces of $L$ do not in general coincide with the subspaces closed as members of $X_{L}^{\circ}$. However it is obvious from the definitions concerned that subspaces of $L$ closed in the latter sense are precisely what Banach [2, p. 117] calls the regularly closed subspaces of $L$. Thus the closure in linear systems includes as special cases both the ordinary topological closure in normed linear spaces and the regular closure of Banach in conjugate spaces. The theorems of this chapter may now be interpreted in an obvious fashion as theorems about normed linear spaces and their closed and regularly closed subspaces. Corollary 1 to Theorem III-10, for example, yields the theorem that any two closed subspaces of a normed linear space which have deficiency one are mutually isomorphic as normed linear spaces. In this connection it might be pointed out that whether or not the linear system of a nonfinite-dimensional normed linear space can fail to be stable does not appear to be known.

In the case of the material of $\S 4$ there are several things to be said about

${ }^{(13)}$ With respect to the usual conjugate space norm topology. 
normed linear spaces which are not mere translations of theorems about general linear systems. We conclude this chapter with a discussion of these.

Lemma. Let $X$ be a normed linear space, let $X_{L}$ be its linear system and let $M$ and $N$ be any two closed disjoint subspaces of $X$. Then $M$ and $N$ are completely disjoint subspaces of $X_{L}$ if and only if there exists a positive real number $A$ such that whenever $x$ is in $M, y$ is in $N$, and $\|x\|=1$ then $\|x-y\| \geqq A$.

Proof. Let $E$ be the unique linear transformation of $M+N$ into itself which coincides on $M$ with the identity and on $N$ with the identically zero transformation. By Theorem III-9, $M$ and $N$ are completely disjoint if and only if $E$ is a homomorphism of $(M+N)_{L}$ into itself and by Theorem II-4 this is the case if and only if $E$ is continuous. But $E$, being linear, is continuous if and only if there exists a positive real number $1 / A$ such that $\|E(x-y)\| \leqq(1 / A)\|x-y\| ;$ that is, $\|x\| \leqq(1 / A)\|x-y\|$ for all $x$ in $M$ and all $y$ in $N$ and this is equivalent to the condition in question.

In this form complete disjointness has been introduced $\left({ }^{14}\right)$ independently by Kober [11] and by Lorch [14], both of whom prove that if $M$ and $N$ are disjoint closed subspaces of a complete normed linear space then $M$ and $N$ are completely disjoint if and only if $M+N$ is closed. In the light of the theory of $\$ 4$ and the theorem of [8] to the effect that the quotient space of a complete normed linear space modulo a closed subspace is again complete, the theorem of Kober and Lorch is seen to be equivalent to the following.

ThEOREM III-13. In the lattice of closed subspaces of a complete normed linear space modularity and d-modularity are equivalent.

We now show by an example that even in the linear systems of normed linear spaces modularity and $d$-modularity need not be equivalent and hence, in particular, that the hypothesis of completeness in Theorem III-13 may. not be dispensed with. Let $X$ be any normed linear space which contains two infinite-dimensional completely disjoint closed subspaces $M$ and $N$, for example, the direct product of any infinite-dimensional Banach space with itself. Let $M_{1}$ and $N_{1}$ be dense proper subspaces of $M$ and $N$ respectively and let $x$ and $y$ be elements of $X$ such that $x \in M-M_{1}$ and $y \in N-N_{1}$. Finally let $Y$ be the incomplete normed linear space obtained by relativizing the topology of $X$ to $M_{1} \dot{+} N_{1} \dot{+}(x+y)$. It is readily verified that $M_{1}$ and $N_{1}$ are closed in $Y$ but that $M_{1}+N_{1}$ containing $x+y$ in its closure is not. By the lemma preceding Theorem III-13 it is clear that $M_{1}$ and $N_{1}$ are completely disjoint. Thus in the lattice of the linear system of $Y, M_{1}$ and $N_{1}$ form a modular pair but not a $d$-modular pair. On the other hand, $M_{1} \dot{+}(x+y)$ and $N_{1}$ trivially form a $d$-modular pair which on account of the preceding remark

${ }^{(14)}$ But not with this terminology. Kober doesn't use any terminology and Lorch omits the word "complete." 
cannot be a stable $d$-modular pair and hence cannot be a modular pair.

We do not know whether such pairs exist in every non-complete normed linear space and hence we do not know whether or not the equivalence of modularity and $d$-modularity is equivalent to completeness in normed linear spaces.

We conclude this chapter with a lemma which when combined with the foregoing has two interesting theorems as consequences. This lemma is due jointly to P. Erdös, S. Kakutani, and the author.

LEMMA. Every infinite-dimensional normed linear space $X$ contains two disjoint closed subspaces which are not completely disjoint.

Proof. There is clearly no loss in generality in supposing that $X$ is separable. In this case it follows from Theorème 4 on page 124 of [2] that there exists a total sequence $\left\{l_{n}\right\}$ of members of $L$ where $X_{L}$ is the linear system of $X$. We may suppose that the $l_{n}$ are linearly independent. For each $k=1,2, \cdots$ let $A_{k}$ be the intersection of the null spaces of $l_{1}, l_{2}, \cdots, l_{2 k}$. It is clear that for each $k=1,2, \cdots, A_{k+1}$ has deficiency two in $A_{k}$. Let $R_{k}$ be a complement of $A_{k+1}$ in $A_{k}$ and let $x_{k}$ and $y_{k}$ be linearly independent members of $R_{k}$ such that $\left\|x_{k}\right\|=\left\|y_{k}\right\|=1$ and $\left\|x_{k}-y_{k}\right\|<1 / k$. Finally let $M$ be the closure of $x_{1}+x_{2}+\cdots$ and let $N$ be the closure of $y_{1}+y_{2}+\cdots$. Suppose that $z$ is any member of $M \cap N$ and that $k=1,2, \ldots$ It follows from Theorem III-1 that $M=x_{1}+x_{2} \dot{+} \cdots+x_{k-1} \dot{+}\left(\right.$ closure of $\left.\left(x_{k}+x_{k+1} \dot{+} \cdots\right)\right)$ and that $N=y_{1} \dot{+} y_{2} \dot{+} \cdots+y_{k-1} \dot{+}\left(\right.$ closure of $\left.\left(y_{k} \dot{+} y_{k+1} \dot{+} \cdots\right)\right)$. Thus $z=c_{1} x_{1}$ $+c_{2} x_{2}+\cdots+c_{k-1} x_{k-1}+x=d_{1} y_{1}+d_{2} y_{2}+\cdots+d_{k-1} y_{k-1}+y$ where the $c$ 's and $d$ 's are real numbers and $x$ and $y$ are in $A_{k}$. Since the $x_{i}$ and $y_{j}$ are linearly independent and $\left(x_{1}+x_{2} \dot{+} \cdots+x_{k-1}+y_{1}+y_{2}+\cdots+y_{k-1}\right) \cap A_{k}=0$ it follows that $c_{i}=d_{j}=0$ for $i, j=1,2, \cdots, k-1$ and hence that $z \in A_{k}$. In other words, $l_{n}(z)=0$ for all $n=1,2, \cdots$ and since $\left\{l_{n}\right\}$ is total, $x=0$. Thus $M$ and $N$ are disjoint and because of the relations $\left\|x_{k}\right\|=\left\|y_{k}\right\|=1$ and $\left\|x_{k}-y_{k}\right\|<1 / k$ are not completely disjoint.

THEOREM III-14. Every infinite-dimensional normed linear space contains two closed subspaces whose linear union is not closed $\left({ }^{15}\right)$.

THEOREM III-15. The lattice of closed subspaces of a normed linear space is modular if and only if the space is finite-dimensional.

\section{Chapter IV. Bounded closure and convergence}

1. Definition and elementary properties of bounded closure. Let $X$ be a linear space and let $L$ be a subspace of $X^{*}$. If $L$ is a norm set, that is, if $X_{L}$ is the linear system of a normed linear space, then it follows from a theo-

(15) That such pairs of subspaces exist in complex Hilbert space has been proved by Stone $[23$, p. 21]. 
rem( $\left.{ }^{16}\right)$ of Banach $\left[2\right.$, p. 80] that a subset $A$ of $X$ is norm bounded( $\left.{ }^{17}\right)$ if and only if for each $l$ in $L$, l.u.b. $x_{x \in A}|l(x)|<\infty$. In any case we shall call an $A$ with the latter property an $L$ bounded subset of $X$ or a bounded subset of $X_{L}$. It is clear that a subset $A$ of $X$ is $L$ bounded if and only if every countable subset of $A$ is $L$ bounded.

By definition every member of $L$ transforms every $L$ bounded subset of $X$ into a bounded set of real numbers. We call the set of all members of $X^{*}$ which do likewise the bounded closure $\bar{L}$ of $L$. It is clear that $L \subseteq \bar{L}$ and that $\bar{L}$ is a subspace. If $L=\bar{L}$ we say that $L$ is boundedly closed and that $X_{L}$ is a boundedly closed linear system. It is obvious that norm sets are boundedly closed and as we shall see below nonboundedly closed $L$ 's exist in great variety whenever $X$ is infinite-dimensional.

If $L$ and $M$ are subspaces of $X^{*}$ it is obvious that $L \subseteq M$ implies $\bar{L} \subseteq \bar{M}$. It is also obvious that $L$ and $\bar{L}$ define the same bounded sets so that $\bar{L}$ is always boundedly closed. It follows that $\bar{L}$ may be characterized as the smallest boundedly closed subspace containing $L$ and that $L$ and $M$ define the same bounded sets in $X$ if and only if $\bar{L}=\bar{M}$.

If $\left\{L_{\alpha}\right\}$ is an arbitrary family of boundedly closed subspaces of $X^{*}$ and $L=\prod L_{\alpha}$ then since, for each $\alpha, L_{\alpha} \supseteq \prod L_{\alpha}$ it follows that $\bar{L}_{\alpha}=L_{\alpha} \supseteq \prod \bar{L}_{\alpha}$. Thus $\prod \bar{L}_{\alpha} \subseteq \prod L_{\alpha}$ so that $\prod L_{\alpha}$ is boundedly closed. In other words the intersection of the members of any family of boundedly closed subspaces of $X^{*}$ is again boundedly closed.

As the reader may verify without difficulty the bounded closure $\left({ }^{18}\right)$ of the linear system $X_{L} \oplus Y_{M}$ is isomorphic to $X_{\bar{L}} \oplus Y_{\bar{M}}$ so that the direct union of two linear systems is boundedly closed if and only if each factor has this property.

\section{Bounded closure and closure.}

THEOREM IV-1. If $X$ is a linear space then every closed subspace $L$ of $\left(X^{*}\right)_{X}$ is boundedly closed. The converse is true if and only if $X$ is finite-dimensional.

Proof. Let $x$ be any nonzero element in $X$ and let $L_{x}$ be the set of all $l$ in $X^{*}$ such that $l(x)=0$. Then the set $\{x, 2 x, 3 x, \cdots\}$ is a bounded subset of $X_{L_{x}}$ while for every $m$ in $X^{*}-L_{x}$ the set $\{m(x), m(2 x), m(3 x), \cdots\}$ is an unbounded set of real numbers. Hence $L_{x}$ is boundedly closed. Since every closed $L$ is an intersection of $L_{x}$ 's the first statement of the theorem follows. To verify the second we observe that if $X$ is finite-dimensional then every subspace of $\left(X^{*}\right)_{X}$ is closed and that if $X$ is infinite-dimensional every norm

(16) Cf. footnote 7.

(17) More generally if $X_{L}$ is the linear system of a convex topological linear space then this statement holds with norm boundedness replaced by the notion of boundedness in general topological linear spaces introduced by Kolmogoroff [12] and von Neumann [21].

(18) By the bounded closure of the linear system $X_{L}$ we mean of course the linear system $X_{L}$. 
set $\left.{ }^{19}\right)$ is an example of a nonclosed but boundedly closed subspace of $\left(X^{*}\right)_{X}$.

TheOREM IV-2. If $L$ is a boundedly closed subspace of $\left(X^{*}\right)_{X}$ then the deficiency of $L$ in its closure is either zero or at least $C$.

Proof. Let $X_{1}$ be a complement of $L^{\prime}$ in $X$.

(Case I-Every $L$ bounded subset of $X_{1}$ is contained in a finite dimensional subspace of $X_{1}$.) Then, since in a regular finite-dimensional linear system every linear functional is distinguished, it follows that every member of $X^{*}$ takes bounded subsets of $X_{1}$ into bounded sets of real numbers. Hence $L^{\prime \prime}$ is contained in and hence equal to $\bar{L}$. Thus since $L=\bar{L}, L$ is closed and its deficiency in its closure is zero.

(Case II-There exists an $L$ bounded sequence $x_{1}, x_{2}, \ldots$ of linearly independent members of $X_{1}$.) Then $l\left(x_{1}\right), l\left(x_{2}\right), \ldots$ is bounded for all $l$ in $L$ while for every sequence of real numbers $\gamma_{1}, \gamma_{2}, \ldots$ there exists $l$ in $L^{\prime \prime}$ with $l\left(x_{n}\right)=\gamma_{n}$ for $n=1,2, \ldots$. It follows that the deficiency of $L$ in $L^{\prime \prime}$ must be at least as great as the deficiency of the space of bounded sequences in the space of all real sequences. That this is $C$ is at once apparent from a consideration of the sequences of the form $1^{x}, 2^{x}, 3^{x}, \ldots$ where $x$ is a positive real number.

CoRollary 1. For subspaces of $\left(X^{*}\right)_{X}$ of deficiency less than $C$, closure and bounded closure are equivalent.

Since boundedly closed subspaces of deficiency one in $\left(X^{*}\right)_{X}$ are closed so are intersections of such subspaces. Thus not every boundedly closed subspace of $\left(X^{*}\right)_{X}$ is an intersection of boundedly closed subspaces of deficiency one. Hence not only do the boundedly closed subspaces of $\left(X^{*}\right)_{X}$ fail to coincide with the closed subspaces of $\left(X^{*}\right)_{X}$ but it is impossible to select a subspace $Y$ of $X^{* *}$ so that the boundedly closed subspaces of $\left(X^{*}\right)_{X}$ coincide with the closed subspaces of $\left(X^{*}\right)_{Y}$.

3. Convergence. For many purposes it is convenient to be able to express the fact that $X_{L}$ is boundedly closed directly in terms of the bounded subsets and closed subspaces of $X_{L}$ without explicit reference to the members of $L$. This may be done as follows. Let $l$ be a nonzero member of $X^{*}$, let $X_{1}$ be the null space of $l$ and let $x$ be an element in $X-X_{1}$. Then $l$ is not in the bounded closure of $L$ if and only if there exists a sequence $\left\{x_{n}\right\}$ of elements of $X_{1}$ and a sequence $\left\{\nu_{n}\right\}$ of real numbers, $n=1,2, \cdots$, such that $\left\{x_{n}-\nu_{n} x\right\}$ is $L$ bounded and $\left\{\nu_{n}\right\}=\left\{l\left(x_{n}-\nu_{n} x\right) / l(-x)\right\}$ tends to infinity. In other words, putting $\gamma_{n}=1 /\left|\nu_{n}\right|$ and $y_{n}=x_{n} / \nu_{n}$ we see that $l$ is not in the bounded closure of $L$ if and only if there exists a sequence $y_{n}$ of elements of $X_{1}$ and a convergent to zero sequence of positive real numbers $\left\{\gamma_{n}\right\}$ such that $\left\{\left(y_{n}-x\right) / \gamma_{n}\right\}$ is $L$ bounded. Now, as is readily verified, if $y_{1}, y_{2}, \cdots ; x$ are elements in a normed

(19) A norm may be defined in any linear space as follows. Let $\left\{\phi_{\alpha}\right\}$ be a Hamel basis for the space and let $\left\|c_{1} \phi \alpha_{1}+\cdots+c_{n} \phi \alpha_{n}\right\|=\left|c_{1}\right|+\cdots+\left|c_{n}\right|$. 
linear space then $\left\|y_{n}-x\right\| \rightarrow 0$ if and only if there exists a sequence $\gamma_{n}$ of positive real numbers tending to zero such that $\left\{\left\|\left(y_{n}-x\right) / \gamma_{n}\right\|\right\}$ is bounded. Thus we are led to make the definition. If $\left\{y_{n}\right\}, n=1,2, \cdots$, is a sequence of elements in a linear system $X_{L}$ and $x$ is an element in $X_{L}$ then $y_{n}$ converges $\left({ }^{20}\right)$ to $x$ or $y_{n} \rightarrow x$ or $x$ is a limit of the sequence $\left\{y_{n}\right\}$ if and only if there exists a convergent to zero sequence of positive real numbers $\gamma_{1}, \gamma_{2}, \ldots$ such that $\left\{\left(y_{n}-x\right) / \gamma_{n}\right\}$ is a bounded subset of $X_{L}$. If $A$ is a subset of $X_{L}$ then the first derivative $A^{(1)}$ of $A$ is the set of all limits of convergent sequences of elements of $A$ and $A$ is sequentially closed if $A^{(1)}=A$. Returning to our discussion of bounded closure we see that our condition may now be phrased as follows. $l$ is not in the bounded closure of $L$ if and only if the first derivative of the null space of $l$ is $X$. Since the first derivative of a subspace is clearly again a subspace we may state the following.

THEOREM IV-3. Let $X_{L}$ be a linear system. Then a member $l$ of $X^{*}$ is in the bounded closure of $L$ if and only if its null space is sequentially closed. In particular $X_{L}$ is boundedly closed if and only if every sequentially closed subspace of deficiency one is closed.

The notion of convergence which we have just introduced turns out to be a rather useful tool not only in the study of bounded closure but for other things as well. Its most important elementary properties are stated in the following theorem whose proof we leave to the reader.

THEOREM IV-4. Let $X_{L}$ be a linear system, let $x, y, x_{1}, x_{2}, \cdots ; y_{1}, y_{2}, \cdots$ be elements of $X$, let $\lambda, \mu, \lambda_{1}, \lambda_{2}, \cdots$ be real numbers and let $A$ be a subset of $X$. Then: (1) If $x_{n} \rightarrow x$ and $y_{n} \rightarrow y$ then $\lambda x_{n}+\mu y_{n} \rightarrow \lambda x+\mu y$. (2) If $\lambda_{n} \rightarrow 0$ and $\left\{x_{n}\right\}$ is bounded then $\lambda_{n} x_{n} \rightarrow 0$. (3) $x_{n} \rightarrow x$ and $x_{n} \rightarrow y$ implies $x=y$ if and only if $X_{L}$ is regular. (4) If $A$ is bounded then $A^{(1)}$ is bounded. (5) If $A$ is convex then $A^{(1)}$ is convex. (6) Every closed subspace of $X_{L}$ is sequentially closed.

Somewhat less trivial is:

TheOREM IV-5. Let $M$ be a subspace of the linear system $X_{L}$ and let $\phi$ be an element of $X_{L}$. Then $(M+\dot{+} \phi)^{(1)}=M^{(1)} \dot{+} \phi$.

Proof. It is sufficient to show that $M^{(1)} \dot{+} \phi \supseteq(M \dot{+} \phi)^{(1)}$. Let $\psi$ be any element in $(M \dot{+} \phi)^{(1)}$. Then there exists a sequence $x_{1}, x_{2}, \ldots$ of elements of $M$ and a sequence $\lambda_{1}, \lambda_{2}, \cdots$ of real numbers such that $x_{n}+\lambda_{n} \phi \rightarrow \psi$. By choosing a subsequence if necessary we may suppose that either $\lambda_{n} \rightarrow \bar{\lambda}$ or $\left|\lambda_{n}\right| \rightarrow \infty$. In the first case we have $\lambda_{n} \phi \rightarrow \bar{\lambda} \phi$ and hence $x_{n} \rightarrow \psi-\bar{\lambda} \phi$. Thus $\psi-\bar{\lambda} \phi \in M^{(1)}$ so that $\psi \in M^{(1)}+\phi$. In the second case we have, on putting $y_{n}=x_{n} /\left(-\lambda_{n}\right)$, $y_{n} \rightarrow \phi$. Thus there exist sequences $\gamma_{1}, \gamma_{2}, \cdots$ and $\mu_{1}, \mu_{2}, \cdots$ of positive real numbers which converge to zero and positive real valued functions $F$ and $G$

(20) Fichtenholz [6] introduces a notion of convergence equivalent to this. 
such that for all $l$ in $L,\left|l\left(x_{n}+\lambda_{n} \phi-\psi\right)\right| \leqq \gamma_{n} F(l)$ and $\left|l\left(y_{n}-\phi\right)\right| \leqq \mu_{n} G(l)$. For each $n=1,2, \cdots$ choose $k_{n}$ so that $\mu_{k_{n}} \leqq \gamma_{n} /\left|\lambda_{n}\right|$. Then we have $\left|l\left(x_{n}+\lambda_{n} y_{k_{n}}-\psi\right)\right| \leqq\left|l\left(x_{n}+\lambda_{n} \phi-\psi\right)\right|+\left|l\left(\lambda_{n} y_{k_{n}}-\lambda_{n} \phi\right)\right| \leqq \gamma_{n} F(l)+\lambda_{n} \mu_{k_{n}} G(l)$ $\leqq \gamma_{n}(F(l)+G(l))$. Hence $\left\{\left(x_{n}+\lambda_{n} y_{k_{n}}-\psi\right) / \gamma_{n}\right\}$ is bounded so that $x_{n}+\lambda_{n} y_{k_{n}} \rightarrow \psi$ and $\psi$ is in $M^{(1)} \subseteq M^{(1)}+\phi$.

4. Stability of bounded closure. By making use of the notion of convergence and the theorems of the preceding section it is possible to obtain some results concerning the effect of small changes in $X$ and $L$ on the bounded closure of $X_{L}$. We begin with a lemma which is a slight generalization of part of Theorem IV-3.

Lemma. If $X_{L}$ is a boundedly closed linear system and $M$ is a subspace of $X$ of finite deficiency such that $M^{(1)}=M$ then $M$ is closed.

Proof. It follows from Theorem IV-5 that every subspace of $X$ which contains $M$ is sequentially closed. But $M$ is an intersection of subspaces of deficiency one and hence by Theorem IV-3 an intersection of closed subspaces. Hence $M$ is closed.

TheOREM IV-6. If $X_{L}$ is a boundedly closed linear system and $M$ is a subspace of $X_{L}$ of finite deficiency then $M_{L}$ is boundedly closed.

Proof. Let $N$ be any subspace of $M$ having deficiency one in $M$. It follows from Theorem IV-5 that $N^{(1)}$ is sequentially closed and hence, by the above lemma, is closed. Hence if $N^{(1)} \cap M=N$ then $N$ is the intersection with $M$ of a closed subspace of $X_{L}$. In other words if $N$ is sequentially closed with respect to $M_{L}$ then $N$ is closed with respect to $M_{L}$. Hence by Theorem IV-3, $M_{L}$ is boundedly closed.

THEOREM IV-7. Let $X_{L}$ be a linear system, let $M_{L}$ be a boundedly closed subsystem, let $N_{L}$ be a second subsystem and let $\phi$ be an element of $X_{L}$. Then if $M \subseteq N \subseteq M^{(1)}, N_{L}$ is boundedly closed. Furthermore the system $(M+\phi)_{L}$ is boundedly closed if and only if either $\phi$ is in $M^{(1)}$ or $\phi$ is not in the closure of $M$.

Proof. Suppose $M \subseteq N \subseteq M^{(1)}$ and let $K$ be any subspace of $N$ of deficiency one which is sequentially closed in $N_{L}$. Then $K \cap M$ is sequentially closed in $M_{L}$ and hence, since $M_{L}$ is boundedly closed, is closed in $M_{L}$. On the other hand $K \subseteq(K \cap M)^{(1)}$. In fact since $K$ is sequentially closed in $N_{L},(K \cap M)^{(1)} \cap N \subseteq K$, and application of Theorem IV-5 tells us that $(K \cap M)^{(1)} \cap N$ has deficiency in $N$ of not more than one. It follows that the closure of $K \cap M$ relative to $\dot{N}_{L}$ contains $K$. Since $K \cap M$ is closed relative ro $M_{L}$ this closure cannot be the whole of $N$ and hence must be $K$. Thus $K$ is closed and by Theorem IV-3, $N_{L}$ is boundedly closed. This completes the proof of the first half of the theorem and allows us to conclude in particular that if $\phi \in M^{(1)}$ then $(M+\phi)_{L}$ is boundedly closed. If $\phi$ is not in the closure of $M$ then $(M \dot{+} \phi)_{L}$ is isomorphic to $M_{L} \oplus(\phi \dot{+})_{L}$, and the latter being the 
direct union of two boundedly closed linear systems is boundedly closed. Finally if $\phi$ is in the closure of $M$ but not in $M^{(1)}$ then $M$ is a nonclosed but sequentially closed subspace of $(M \dot{+} \phi)_{L}$. Thus by Theorem IV-3, $(M \dot{+} \phi)_{L}$ is not boundedly closed.

TheOREM IV-8. If $X$ is a linear space and $L$ is a boundedly closed subspace of $X^{*}$ then, whenever $M$ is a finite-dimensional subspace of $X^{*}, L+M$ is boundedly closed.

Proof. It is sufficient to show that $L+l$ is boundedly closed whenever $l$ is an element of $X^{*}-L$. Let $X_{1}$ be the null space of $l$. Let $\phi$ be any element in $X-X_{1}$. Now $\left(X_{1}\right)_{L+i}=\left(X_{1}\right)_{L}$ and by Theorem IV-6 the latter is boundedly closed. Hence, since $\phi \notin X_{1}$ and $X_{1}$ is closed in $\mathrm{X}_{(L+\ell)}$, it follows from Theorem IV-7 that $X_{(L+\dot{+})}$ is boundedly closed.

It follows from Theorem IV-1 and the corollary to Theorem III-1 that every finite-dimensional subspace of $\left(X^{*}\right)_{X}$ is boundedly closed. We shall show later that every $\boldsymbol{\aleph}_{0}$-dimensional subspace of $\left(X^{*}\right)_{X}$ is boundedly closed. Whether or not Theorem IV-8 remains true when $M$ is a general boundedly closed subspace of $X^{*}$ or even an $\boldsymbol{\aleph}_{0}$-dimensional subspace of $X^{*}$ is unknown. The best result we have along these lines will appear in the next chapter in connection with our discussion of "the first countability property."

Theorem IV-9. Let $X$ be a linear space, let $L$ be a total boundedly closed subspace of $\left(X^{*}\right)_{X}$ and let $M$ be a subspace of $\left(X^{*}\right)_{x}$ such that $M \subseteq L$ and $L / M$ is one-dimensional. Then $M$ is boundedly closed if and only if $M$ is the null space of a member of $\left(L^{*}\right)_{L}$ which is in the first derivative of $X$.

Proof. Let $f$ be a nontrivial member of $L^{*}$ which vanishes on $M$. If $X_{M}$ is boundedly closed then $(X \dot{+} f)_{M}$, being isomorphic either to $X_{M} \oplus(f \dot{+})_{M}$ or to $X_{M}$ is also boundedly closed. Hence by Theorem IV-8 $(X+f)_{L}$ is boundedly closed. Hence by Theorem IV-7, since $f$ is obviously in the closure of $X$, $f$ is in the first derivative of $X$. Conversely if $f$ is in the first derivative of $X$ then $(X+f)_{L}$ is boundedly closed. But $M$ is a closed subspace of the conjugate of $(X \dot{+} f)_{L}$. Hence $(X \dot{+} f)_{M}$ is boundedly closed. Hence, by Theorem IV-6, $X_{M}$ is boundedly closed.

5. Completeness. Let $X_{L}$ be a regular linear system. Then $X_{L}$ may be regarded as a subsystem of $L_{L}^{*}$ and $X$ may or may not be sequentially closed with respect to the convergence in $\left(L^{*}\right)_{L}$. If it is we shall say that the system $X_{L}$ is complete. The intersection of the sequentially closed subspaces of $\left(L^{*}\right)_{L}$ which contain $X$ we shall call the completion of $X_{L}$. It is obvious that the completion of $X_{L}$ is complete. Thus every regular linear system is isomorphic to a subsystem of a complete linear system.

Completeness also may be formulated in terms of "Cauchy sequences" and for some purposes it is convenient to have it so. We call a sequence $x_{1}, x_{2}, \cdots$ of elements of a linear system $X_{L}$ a Cauchy sequence if there exists 
an infinite matrix $\left\{\mu_{m, n}\right\}, m, n=1,2, \cdots$ of positive real numbers such that $\mu_{m, n} \rightarrow 0$ as $\left({ }^{21}\right) m$ and $n \rightarrow \infty$ and such that $\left\{\left(x_{n}-x_{m}\right) / \mu_{m, n}\right\}$ is bounded. Then, as the reader may readily verify, a regular linear system $X_{L}$ is complete if and only if whenever $x_{1}, x_{2}, \cdots$ is a Cauchy sequence of members of $X_{L}$ then there exists $x$ in $X_{L}$ such that $x_{n} \rightarrow x$. It follows immediately that a linear system is complete if and only if its bounded closure is complete.

THEOREM IV-10. A regular boundedly closed linear system $X_{L}$ is complete if and only if no total subspace $L_{1}$ of $L$ which has deficiency one in $L$ is boundedly closed.

Proof. The truth of this theorem is an immediate consequence of Theorem IV-9.

It follows from Theorem IV-10 that if $X_{L}$ is a complete boundedly closed linear system and $l$ is an element of $X^{*}-L$ then $X_{(L+l)}$ is not a complete linear system.

6. Completely boundedly closed linear systems. As we have already pointed out the linear system of a normed linear space is always boundedly closed. It follows from Theorem II- 6 that any subsystem being the linear system of a normed linear space is also boundedly closed. As we shall see this is not true of boundedly closed linear systems in general. Hence we introduce the definition: A linear system all of whose subsystems are boundedly closed will be called a completely boundedly closed linear system.

THEOREM IV-11. A linear system $X_{L}$ is completely boundedly closed if and only if for every subspace $M$ of $X, M^{(1)}$ is closed.

Proof. Suppose that $X_{L}$ is completely boundedly closed but that for some subspace $M, M^{(1)}$ is not closed. Then there exists an element $\phi$ which is in the closure of $M^{(1)}$ and not in $M^{(1)}$. But the closures of $M$ and $M^{(1)}$ are identical and since $M_{L}$ and $(M \dot{+} \phi)_{L}$ are boundedly closed this contradicts Theorem IV-7. Conversely suppose that $M^{(1)}$ is closed for all subspaces $M$ of $X_{L}$. Let $N$ be a subspace of $X_{L}$ and let $K$ be a subspace of deficiency one in $N$ sequentially closed with respect to $N_{L}$. Then $N \nsubseteq K^{(1)}$. Hence the closure of $K$ does not contain $N$. Hence $K$ is a closed subspace of $N_{L}$. Thus by Theorem IV-3, $N_{L}$ is boundedly closed.

The question arises as to whether there exist boundedly closed linear systems which are not completely boundedly closed. We answer it in the affirmative by means of a theorem which has certain independent interest.

Theorem IV-12. Let $X$ be a linear space. Then the linear system $\left(X^{*}\right)_{X}$ is boundedly closed if and only if the dimension $\boldsymbol{\aleph}$ of $X$ is such that on an abstract set $S$ of cardinal $\aleph$ there exists no measure $\alpha$ with all of the following properties.

(21) That is, for each $\epsilon>0$ there exist $n_{0}$ and $m_{0}$ such that if $n>n_{0}$ and $m>m_{0}$ then $\mu_{n, m}<\epsilon$. 
(1) $\alpha$ is defined for all subsets of $S$. (2) $\alpha$ is countably additive. (3) $\alpha$ takes on only the values zero and one. (4) $\alpha$ is zero as a function of points. (5) $\alpha(S)=1$.

Proof. Let $\left\{\phi_{\alpha}\right\}$ be a Hamel basis for $X$. Then there is an obvious natural one-to-one linear correspondence between the members of $X^{*}$ and the members of the space $R$ of all real valued functions defined on $\left\{\phi_{\alpha}\right\}$. Under this correspondence members of $X^{* *}$ associated with members of $X$ correspond to the finite linear combinations of point functionals on $R$, where a point functional $F$ is a functional such that for some $\phi_{\alpha}, F(f)=f\left(\phi_{\alpha}\right)$ for all $f$ in $R$. Furthermore it is clear that a subset of $X^{*}$ is bounded as a subset of the linear system $\left(X^{*}\right)_{X}$ if and only if its map in $R$ is a bounded subset of the vector lattice $\left({ }^{22}\right) R$. Thus $\left(X^{*}\right)_{X}$ is boundedly closed if and only if every bounded linear functional on $R$ is a finite linear combination of point functionals. However it is shown in [19] that this last condition is satisfied if and only if $\boldsymbol{N}$ has the measure theoretic property in question. This completes the proof of the theorem.

The existence of measures with properties (1) through (5) of Theorem V-12 has been considered by Ulam [25] and he has shown that whenever $\aleph$ is a cardinal for which no such measure exists then $2^{\mathbb{N}}$ is also such a cardinal. As a consequence we have the following theorem.

THEOREM IV-13. Let $X$ be a linear space rohose dimension is less than $\boldsymbol{N}_{0}+2^{\boldsymbol{N}_{0}}+2^{\left(2^{\left.\boldsymbol{N}_{0}\right)}\right.}+\cdots\left(\boldsymbol{N}_{0}\right.$ terms$)$. Then the linear system $\left(X^{*}\right)_{X}$ is boundedly closed.

It is now easy to construct an example of a boundedly closed noncompletely boundedly closed linear system. Let $X_{L}$ be the linear system of a separable Banach space. Then $X$ and $L$ are both $C$-dimensional. Let $f$ be any member of $L^{*}-X$ and let $M$ be the null space of $f$. By Theorem IV-10, $X_{M}$ is not boundedly closed. On the other hand $X_{M}$ is a subsystem of $\left(M^{*}\right)_{M}$ and by Theorem IV-13 $\left(M^{*}\right)_{M}$ is boundedly closed. Thus $\left(M^{*}\right)_{M}$ is boundedly closed but not completely boundedly closed.

\section{Chapter V. Boundedness}

1. Properties of bounded sets. Let $X_{L}$ be a linear system and let $\mathscr{B}$ be the family of all bounded subsets of $X_{L}$. For any subset $A$ of $X$ let $\operatorname{Co}(A)$ denote the smallest convex subset of $X$ which contains $A$ and let $\tilde{C} o(A)$ denote the smallest convex subset of $X$ which contains $A$ and contains $-x$ with $x$. For any two subsets $A_{1}$ and $A_{2}$ of $X$ and any two real numbers $\lambda_{1}$ and $\lambda_{2}$ let $\lambda_{1} A_{1}+\lambda_{2} A_{2}$ denote the set of all elements in $X$ of the form $\lambda_{1} x_{1}+\lambda_{2} x_{2}$ where $x_{1}$ is in $A_{1}$ and $x_{2}$ is in $A_{2}$. The following statements are obvious consequences of the definitions concerned and will be used freely in the sequel. (1) If $A$ is a subset of $X$ containing only a finite number of elements then

(22) See chap. VII of [3] for a discussion of this concept. 
$A$ is in B. (2) If $A_{1}$ is in $\mathbb{B}$ and $A_{2}$ is a subset of $A_{1}$ then $A_{2}$ is in B. (3) If $A_{1}$ and $A_{2}$ are in $\mathcal{B}$ and $\lambda_{1}$ and $\lambda_{2}$ are real numbers then $\lambda_{1} A_{1}+\lambda_{2} A_{2}$ and $A_{1} \cup A_{2}$ are in $B$. (4) If $A$ is in $\mathbb{B}$ then $C o(A)$ and $\tilde{C} o(A)$ are in $\mathscr{B}$. (5) If $A$ is a subset of $X$ which is not in $\mathbb{B}$ then there is a countable subset of $A$ which is not in $\mathbb{B}$. (6) If $A$ is in $B$ then $A^{(1)}$ is in $B$.

We remark that these properties do not serve to characterize families of bounded sets in linear systems. In fact, as the reader may readily verify, if we let $X$ be a normed linear space and let $B$ be the family of all subsets of $X$ with compact closures then statements (1) through (6) are all valid. On the other hand, the members of $X^{*}$ which carry the members of $B$ into bounded sets of real numbers are precisely the continuous linear functionals while, as is well known, the unit sphere in $X$ does not lie in $B$. It follows that the members of $\mathbb{B}$ are not the bounded sets of $X_{L}$ for any choice of $L$ in $X^{*}$.

2. Five types of boundedness. In this section we introduce a classification of linear systems according to the nature of their families of bounded sets. This classification in a sense supplements that of the last chapter. There we were interested only in whether a linear system was boundedly closed or not. Here we shall study properties which are independent of the relationship of the system to its bounded closure and in fact are possessed by the system if and only if possessed by its bounded closure.

Let $X_{L}$ be a linear system. If there exists a bounded subset $A$ of $X_{L}$ such that every other bounded subset of $X_{L}$ is a subset of some $\lambda A$ where $\lambda$ is a real number we say that $X_{L}$ is simple. If there exists a countable family $A_{1}, A_{2}, \cdots$ of bounded subsets of $X_{L}$ such that every other bounded subset of $X_{L}$ is a subset of some $A_{i}$ we say that $X_{L}$ has the second countability property. If whenever $A_{1}, A_{2}, \cdots$ is a countable family of bounded subsets of $X_{L}$ there exists a sequence $\gamma_{1}, \gamma_{2}, \ldots$ of positive real numbers such that $\left(A_{1} / \gamma_{1}\right) \cup\left(A_{2} / \gamma_{2}\right) \cup \ldots$ is a bounded subset of $X_{L}$ we say that $X_{L}$ has the first countability property. If there exists a bounded subset of $X_{L}$ whose linear span is $X$ we say that $X_{L}$ is relatively bounded $\left({ }^{23}\right)$. If there exists a countable family $A_{1}, A_{2}, \cdots$ of bounded subsets of $X_{L}$ such that the linear span of $A_{1} \cup A_{2} \cup \ldots$ is $X$ we say that $X_{L}$ is almost relatively bounded.

THEOREM V-1. Let $X_{L}$ be a linear system. If it has the second countability property or is relatively bounded then it is almost relatively bounded. If it is simple it is relatively bounded and has both countability properties. If it has both countability properties then it is simple. If it is almost relatively bounded and has the first countability property then it is relatively bounded.

Proof. The proof is obvious.

Theorem V-1 tells us that of the $2^{5}$ disjoint classes into which our five

(23) The choice of this term stems from the fact that $X_{L}$ is relatively bounded if and only if $L$ is a "sufficiently small" subspace of $X^{*}$. 
properties divide linear systems, at most eight are not empty. These are: (1) The simple linear systems. (2) The relatively bounded linear systems having the first countability property and not the second. (3) The relatively bounded linear systems having the second countability property and not the first. (4) The relatively bounded linear systems having neither countability property. (5) The nonrelatively bounded linear systems having the second countability property. (6) The almost relatively bounded nonrelatively bounded linear systems with neither countability property. (7) The non almost relatively bounded linear systems having the first countability property. (8) The non almost relatively bounded linear systems with neither countability property. We shall give examples in the last chapter showing that none of these classes is empty and hence that Theorem V-1 cannot be strengthened.

TheOREM V-2. Let $X_{L}$ be a linear system and let $Y$ be a subspace of $X$. Then whenever $X_{L}$ has one of the five properties listed at the beginning of this section, $Y_{L}$ also has this property.

Proof. The proof is obvious.

Theorem V-3. Let $X_{L}$ and $Y_{M}$ be linear systems. Let $P$ represent any one of the five properties under discussion. Then $X_{L} \oplus Y_{M}$ has property $P$ if and only if both $X_{L}$ and $Y_{M}$ have property $P$.

Proof. This theorem is an immediate consequence of Theorem V-2 and the following lemma.

Lemma. If $X_{L}$ and $Y_{M}$ are linear systems then a subset $C$ of $X_{L} \oplus Y_{M}$ is bounded if and only if there exist bounded subsets $A$ and $B$ of $X_{L}$ and $Y_{M}$ respectively such that $C \subseteq A+B$.

Proof. Half of the lemma is obvious. If $C$ is a bounded subset of $X_{L} \oplus Y_{M}$ let $A$ be the set of all $x$ in $X$ such that for some $y$ in $Y, x+y$ is in $C$ and let $B$ be defined similarly with respect to $Y$. Obviously $A$ and $B$ are bounded and $C \subseteq A+B$.

\section{Relative boundedness.}

THEOREM V-4. Let $X_{L}$ be a relatively bounded linear system and let $M$ be a subspace of $X$. Then the linear systems $X_{L} / M$ and $X_{L}^{\circ}$ are both relatively bounded.

Proof. The first statement is obvious. Let $A$ be a bounded subset of $X_{L}$ whose linear span is $X$. Let $A^{\circ}$ be the set of all $l$ in $L$ such that l.u.b. $x_{x \in A}|l(x)|<1$. Since the linear span of $A$ is $X, A^{\circ}$ is a bounded subset of $X_{L}^{\circ}$ and since $A$ is a bounded subset of $X_{L}, A^{\circ}$ has $L$ for its linear span. Thus $X_{L}^{\circ}$ is relatively bounded. 
THEOREM V-5. Let $X$ be a linear space. Let $L$ and $M$ be subspaces of $\left(X^{*}\right)_{X}$. If $L$ is relatively bounded and $M \subseteq L$ then $M$ is relatively bounded. If $L$ and $M$ are relatively bounded then $L+M$ is relatively bounded. If $L$ is finitedimensional or a norm set then $L$ is relatively bounded.

Proof. The first statement is obvious. Suppose that $L$ and $M$ are relatively bounded. Let $A$ and $B$ be bounded subsets of $X_{L}$ and $X_{M}$ respectively whose linear spans are $X$. Then it is clear that $\tilde{C} o(A) \cap \tilde{C} o(B)$ also has $X$ for its linear span and is a bounded subset of $X_{(L \dot{+} M)}$. It is obvious that every onedimensional subspace of $\left(X^{*}\right)_{X}$ is relatively bounded and hence by what we have just proved every finite-dimensional subspace is also. Norm sets are obviously relatively bounded.

That $\boldsymbol{\aleph}_{0}$-dimensional subspaces of $\left(X^{*}\right)_{X}$ need not be relatively bounded will appear below.

THEOREM V-6. If $X$ is a linear space then $X^{*}$ is relatively bounded if and only if $X$ is finite-dimensional.

Proof. That $X^{*}$ is relatively bounded when $X$ is finite-dimensional follows from Theorem V-5. That it is not when $X$ is infinite-dimensional follows from the fact that any bounded subset of $X_{X^{*}}$ must have a finite-dimensional linear span.

Theorem V-7. Let $X$ be a linear space whose dimension is not less than $C$. Then there exists an $\aleph_{0}$-dimensional subspace $L$ of $\left(X^{*}\right)_{x}$ no infinite-dimensional subspace of which is relatively bounded.

Proof. It will be sufficient to consider the case in which the dimension of $X$ is exactly $C$, for in any other case we can choose a $C$-dimensional subspace $M$ and extend the members of the $L$ for this by letting them vanish on some fixed complement of $M$. Furthermore because of the mutual isomorphism of all linear spaces of the same dimension we need only prove the theorem for a particular $C$-dimensional linear space. Let $Y$ be any $\boldsymbol{\aleph}_{0}$-dimensional linear space and consider the linear system $Y_{Y}$. It is obvious that $Y_{Y}$ is isomorphic to all of its infinite-dimensional subsystems. But by Theorem V-6, $Y_{Y^{*}}$ is not relatively bounded. Hence whenever $Y_{1}$ is an infinite-dimensional subspace of $Y,\left(Y_{1}\right)_{Y}$ and hence $\left(Y^{*}\right)_{Y_{1}}$ fails to be relatively bounded. In other words $Y$ is an $\boldsymbol{\aleph}_{0}$-dimensional subspace of $\left(Y^{* *}\right)_{Y} \cdot$ no infinite-dimensional subspace of which is relatively bounded. Since $Y^{*}$ is $C$-dimensional this completes the proof of the theorem.

On the other hand we have:

THEOREM V-8. If $X$ is an $\boldsymbol{\aleph}_{0}$-dimensional linear space then every $\boldsymbol{\aleph}_{0}$-dimensional subspace of $\left(X^{*}\right)_{X}$ is relatively bounded.

Proof. Let $L$ be an $\aleph_{0}$-dimensional subspace of $X^{*}$. Let $L^{\prime}=Y$ and let $Z$ 
be a complement of $Y$ in $X$. Then $X_{L}$ is isomorphic to the linear system $Y_{0} \oplus Z_{L}$ where $Y_{0}$ is the linear system obtained from $Y$ by distinguishing only the zero functional. Because of Theorem V-3 we need only prove that $Y_{0}$ and $Z_{L}$ are relatively bounded. This is obvious for $Y_{0}$. Furthermore since $Z_{L}$ is regular the lemma immediately preceding Theorem III-11 applies and we may infer the existence of Hamel bases $z_{1}, z_{2}, \ldots ; l_{1}, l_{2}, \ldots$ for $Z$ and $L$ respectively such that $l_{i}\left(z_{j}\right)=\delta_{i}^{j}$ for $i, j=1,2, \ldots$. It is obvious that $z_{1}, z_{2}, \ldots$ is a bounded subset of $Z_{L}$ whose linear span is $Z$. Thus $Z_{L}$ is relatively bounded and the theorem is proved.

We conclude this section with a theorem which among other things gives us further information about the "smallness" of relatively bounded subspaces of $\left(X^{*}\right)_{X}$ 's. Preparatory to this we prove a lemma about normed linear spaces.

Lemma. If $X$ is an $\boldsymbol{\aleph}_{0}$-dimensional normed linear space then $X$ contains a dense Hamel basis.

Proof. It is obvious that $X$ is separable. Let $\psi_{1}, \psi_{2}, \ldots$ be a dense countable subset of $X$ such that $\psi_{1} \neq 0$. Define a sequence of elements $\eta_{1}, \eta_{2}, \cdots$ by induction as follows. Let $\eta_{1}=0$. If $\psi_{2}$ is linearly dependent on $\psi_{1}$ choose $\eta_{2}$ linearly independent of $\psi_{1}$ and such that $\left\|\eta_{2}\right\|<1 / 2$; otherwise take $\eta_{2}=0$. If $\eta_{1}, \eta_{2}, \cdots, \eta_{n}$ have been chosen so that $\left\|\eta_{k}\right\|<1 / k$ for $k=1,2, \cdots, n$ and so that $\psi_{1}+\eta_{1}, \psi_{2}+\eta_{2}, \cdots, \psi_{n}+\eta_{n}$ is a linearly independent set of elements, choose $\eta_{n+1}$ as follows. If $\psi_{n+1}$ is not in $\left(\psi_{1}+\eta_{1}\right)+\left(\psi_{2}+\eta_{2}\right) \dot{+} \cdots \dot{+}\left(\psi_{n}+\eta_{n}\right)$, let $\eta_{n+1}=0$. In the contrary case let $\eta_{n+1}$ be any element in the complement of $\left(\psi_{1}+\eta_{1}\right) \dot{+}\left(\psi_{2}+\eta_{2}\right) \dot{+} \cdots \dot{+}\left(\psi_{n}+\eta_{n}\right)$ such that $\left\|\eta_{n+1}\right\|=1 /(n+1)$. It is clear that the sequence of elements $\eta_{1}, \eta_{2}, \ldots$ that we have just defined is such that the sequence $\psi_{1}+\eta_{1}, \psi_{2}+\eta_{2}, \ldots$ is contained in a Hamel basis. It remains to show that it is dense in $X$. Let $x$ be an arbitrary element of $X$ and let $\epsilon$ be an arbitrary positive real number. Let $n_{0}$ be a positive integer such that $1 / n_{0}<\epsilon / 2$. Since a normed linear space contains no isolated points the set $\psi_{n_{0}}, \psi_{n_{0}+1}, \psi_{n_{0}+2}, \cdots$ is also dense in $X$. Let $j$ be a non-negative integer such that $\left\|\psi_{n_{0}+j}-x\right\|<\epsilon / 2$. Then $\left\|x-\left(\psi_{n_{0}+j}+\eta_{n_{0}+j}\right)\right\| \leqq\left\|x-\psi_{n_{0}+j}\right\|+\left\|\eta_{n_{0}+j}\right\|<\epsilon / 2$ $+1 /\left(n_{0}+j\right)<\epsilon / 2+\epsilon / 2=\epsilon$. This completes the proof of the lemma.

TheOREM V-9. Let $X$ be an infinite-dimensional linear space, let $L$ be an arbitrary relatively bounded subspace of $\left(X^{*}\right)_{X}$, and let $M$ be an arbitrary subspace of $L$ which is closed as a subspace of $X_{L}^{\circ}$. Then there exists an automorphism $T^{*}$ of the linear system $\left(X^{*}\right)_{X}$ such that $T^{*}(L) \cap L=M$.

Proof. (Case $\mathrm{I}-X$ is $\boldsymbol{\aleph}_{0}$-dimensional and $M$ is zero-dimensional.) Since $X_{L}$ is relatively bounded there exists a Hamel basis $\phi_{1}, \phi_{2}, \cdots$ for $X$ which is a bounded subset of $X_{L}$. For each $x=c_{1} \phi_{1}+c_{2} \phi_{2}+\cdots+c_{n} \phi_{n}$ in $X$ let $\|x\|=\left|c_{1}\right|+\left|c_{2}\right|+\cdots+\left|c_{n}\right|$. Then $X$ becomes a normed linear space for which all members of $L$ are continuous linear functionals. By the immedi- 
ately preceding lemma there exists a Hamel basis $\theta_{1}, \theta_{2}, \ldots$ which is dense in $X$ with respect to this norm. Let $T$ be the unique automorphism of the linear space $X$ such that $T\left(\phi_{n}\right)=\theta_{n}$ for $n=1,2, \cdots$ and let $T^{*}$ be the unique automorphism of $\left(X^{*}\right)_{X}$ such that $l(T(x))=T^{*}(l)(x)$ for all $l$ in $X^{*}$. We show that $T^{*}(L) \cap L=0$. Let $l$ be any nonzero member of $L$. It will suffice to show that $\left\{T^{*}(l)\left(\phi_{n}\right)\right\}$ is not bounded; that is, that $\left\{l\left(T\left(\phi_{n}\right)\right)\right\}=\left\{l\left(\theta_{n}\right)\right\}$ is not bounded. Let $c$ be any positive real number. Choose $x$ in $X$ so that $l(x)=2 c$ and $n_{0}$ so that $\left\|\theta_{n_{0}}-x\right\|<c /\|l\|$. Then $\left|l\left(\theta_{n_{0}}\right)\right|=\left|l(x)-l(x)+l\left(\theta_{n_{0}}\right)\right| \geqq|l(x)|$ $-\left|l\left(\theta_{n_{0}}\right)-l(x)\right| \geqq|l(x)|-\|l\|\left\|\theta_{n_{0}}-x\right\|>2 c-c=c$. Thus $\left\{l\left(\theta_{n}\right)\right\}$ is not bounded and Case $I$ is disposed of.

(Case II $-X$ is arbitrary but the deficiency of $M$ in $L$ is infinite.) Let $Y=M^{\prime}$ and let $Z$ be a complement of $Y$ in $X$. Since $M=M^{\prime \prime}$ it follows from Theorem III-2 that $M^{\prime}$ is infinite-dimensional. Hence the dimension of $Y$ is $\boldsymbol{N}_{0}$ where $\boldsymbol{N}$ is a cardinal greater than zero. Accordingly we may, by dealing in an obvious manner with a Hamel basis for $Y$, "decompose" $Y$ into $\aleph$ subspaces $X_{\alpha}$, each of which is $\boldsymbol{\aleph}_{0}$-dimensional, such that each element in $Y$ has a unique representation as a sum of elements one from each of the $X_{\alpha}$. For each $\alpha$ let $L_{\alpha}$ be the set of all linear functionals in $\left(X_{\alpha}\right)^{*}$ coinciding on $X_{\alpha}$ with a member of $L$. By Case I there exists an automorphism $T_{\alpha}$ of $X_{\alpha}$ such that $T_{\alpha}{ }^{*}\left(L_{\alpha}\right) \cap L_{\alpha}=0$. Let $T$ be the unique automorphism of $X$ which coincides on $X_{\alpha}$ with $T_{\alpha}$ for each $\alpha$ and on $Z$ with the identity. Let $l$ be any member of $L$ and let $l^{*}=T^{*}(l) . l^{*}$ coincides on each $X_{\alpha}$ with a member of $T_{\alpha}{ }^{*}\left(L_{\alpha}\right)$. Hence if $l^{*} \in L$ then $l^{*}\left(X_{\alpha}\right)=0$ for all $\alpha$. In other words, $l^{*} \in Y^{\prime}=M^{\prime \prime}=M$. Conversely if $l$ is in $M$ then $l(Y)=0$. Thus since $T$ is the identity on $Z$ it follows easily that $T^{*}(l)=l$. Hence $l$ is in $L \cap T^{*}(L)$.

(Case III $-X$ is arbitrary. $M$ has finite deficiency in $L$.) Let $M^{\prime \prime}$ be the closure of $M$ in $\left(X^{*}\right)_{X}$. Then since $M$ is closed in $X_{L}^{\circ}, M^{\prime \prime} \cap L=M$. Thus if $N$ is any complement of $M$ in $L, M^{\prime \prime} \cap N=0$. Furthermore because of Theorem III-1, $L^{\prime \prime}=M^{\prime \prime}+N$. Let $Y$ be a complement of $L^{\prime}$ in $X$. Then $Y^{\prime} \cap L^{\prime \prime}=0$ and $\left(Y^{\prime}+L^{\prime \prime}\right)^{\prime \prime}=X^{*}$. Now since in $X_{X^{*}}$ every subspace is closed it follows that every pair of subspaces is both a modular pair and a $d$-modular pair. Hence in $\left(X^{*}\right)_{X}$ every pair of closed subspaces also has these properties. Thus $Y^{\prime}+L^{\prime \prime}=X^{*}$ and $\left(X^{*}\right)_{X}$ may be regarded as the direct union of the linear systems $\left(M^{\prime \prime}+Y^{\prime}\right)_{X}$ and $N_{X}$. Since $X$ is infinite-dimensional and $L$ is relatively bounded it is clear that there exists a subspace of $M^{\prime \prime}+Y^{\prime}$, of any finite dimension, which is disjoint from $L$ and hence that there is a complement $K$ of $\left(M^{\prime \prime}+Y^{\prime}\right)$ such that $K \cap L=0$. Since $K$ is finite-dimensional it is closed and we see that $\left(X^{*}\right)_{X}$ may also be regarded as the direct union of the linear systems $\left(M^{\prime \prime}+Y^{\prime}\right)_{X}$ and $K_{X}$. Thus it is clear that there is an automorphism $T^{*}$ of $\left(X^{*}\right)_{X}$ such that $T^{*}(l)=l$ for all $l$ in $M^{\prime \prime}+Y^{\prime}$. and such that $T^{*}(N)=K$. Now $T^{*}(L)=T^{*}(M \dot{+} N)=T^{*}(M) \dot{+} T^{*}(N)=M \dot{+} K$ and $(M \dot{+} K)$ $\cap(M+N)=M$. This completes the proof of the theorem. 
4. Almost relative boundedness. The theory of almost relative boundedness differs but little from that of relative boundedness and we shall not develop it in detail. We summarize what we know in a theorem.

TheOREM V-10. If in Theorems V-4 through V-9 the word "almost" is inserted before each occurrence of the words "relatively bounded," then these theorems remain true provided that the following additional changes are made. (1) In Theorem V-4 the second sentence is changed to read "The linear system $X_{L} / M$ is almost relatively bounded." (2) In Theorem V-6 the phrase " $X$ is finite-dimensional" is replaced by the phrase " $X$ has dimension not greater than $\aleph_{0}$." (3) In Theorem V-9 the phrase "Let $X$ be an infinite-dimensional linear space" is replaced by the phrase "Let $X$ be a linear space whose dimension exceeds $\aleph_{0 .}$.

Proof. The truth of this theorem is easily verified using Theorems V-4 through V-9 and we leave the details to the reader.

An example of an almost relatively bounded linear system whose conjugate is not almost relatively bounded is furnished us by $X_{X} \cdot$ whenever $X$ has dimension $\aleph_{0}$.

\section{The first countability property.}

Theorem V-11. Let $X$ be a linear space and let $L_{1}, L_{2}, \cdots$ be a sequence of subspaces of $\left(X^{*}\right)_{X}$ each of which has the first countability property. Then $L=L_{1} \dot{+} L_{2} \dot{+} \cdots$ has the first countability property.

Proof. Let $B_{1}, B_{2}, \cdots$ be a sequence of bounded subsets of the linear system $X_{L}$. For each $i=1,2, \cdots$ and each $j=1,2, \cdots B_{j}$ is a bounded subset of $X_{L_{i}}$. Hence since $X_{L_{i}}$ has the first countability property there exists a sequence of positive real numbers $\gamma_{1}^{\mathbf{q}}, \gamma_{2}^{t}, \ldots$ such that $\left(B_{1} / \gamma_{1}^{\mathbf{q}}\right) \cup\left(B_{2} / \gamma_{2}^{\mathbf{q}}\right) \cup \ldots$ is a bounded subset of $X_{L_{i}}$. For each $k=1,2, \cdots$ let $\nu_{k}$ be the largest of the numbers $\gamma_{k}^{1}, \gamma_{k}^{2}, \cdots, \gamma_{k}^{k}$. Then for each $i=1,2, \cdots$ we have $\gamma_{j}^{\natural} \leqq \nu_{j}$ for $j=i, i+1, i+2, \cdots$. Hence if we let $\mu_{i}$ be the largest of the numbers $\left\{1,\left(\gamma_{1}^{i} / \nu_{1}\right),\left(\gamma_{2}^{1} / \nu_{2}\right), \cdots,\left(\gamma_{i}^{i} / \nu_{i}\right)\right\}$ we shall have $\gamma_{j}^{\}} \leqq \mu_{i} \nu_{j}$ for $i, j=1,2, \cdots$. Thus $\left(B_{1} / \mu_{i} \nu_{1}\right) \cup\left(B_{2} / \mu_{i} \nu_{2}\right) \cup \ldots$ and hence $\left(B_{1} / \nu_{1}\right) \cup\left(B_{2} / \nu_{2}\right) \cup \ldots$ is a bounded subset of $X_{L_{i}}$ for each $i=1,2, \ldots$ It follows that $\left(B_{1} / \nu_{1}\right) \cup\left(B_{2} / \nu_{2}\right)$ $\cup \ldots$ is a bounded subset of $X_{L}$. Thus $X_{L}$ has the first countability property.

Corollary. Every $\aleph_{0}$-dimensional subspace of $\left(X^{*}\right)_{X}$ has the first countability property.

Theorem V-11 tells us that the first countability property is "more stable with respect to increases in size" than relative boundedness. With respect to decreases in size however the situation is quite different. We shall see later that not only may subspaces of $L$ 's with the first countability property fail to have this property but also that countable intersections of such $L$ 's may 
fail to have it. We do not know what the situation is with respect to finite intersections. We can however prove the following.

Theorem V-12. Let $X$ be a linear space and let $L$ be a subspace of $\left(X^{*}\right)_{X}$ having the first countability property. Then every subspace of $L$ having finite deficiency in $L$ also has the first countability property.

For the proof of this theorem as well as the proof of the corresponding theorem concerning the second countability property we need the following lemma.

LEMMA. If $B$ is a bounded subset of the linear system $X_{L}$ and $l$ is a member of $X^{*}$ which is unbounded on $B$, then if $Y$ is the null space of $l, \tilde{C} o(B) \cap Y$ is sequentially dense in $\tilde{C}_{o}(B)$.

Proof. Denote $\tilde{C} o(B)$ by $C$. Let $\phi$ be any member of $C$ which is not in $Y$. For each $n=1,2, \ldots$ choose $x_{n}$ in $C$ such that $l\left(x_{n}\right)=-n l(\phi)$ and let $y_{n}=x_{n}+n \phi$. Then $y_{n}-n \phi \in C$ and since $\phi$ is in $C$ and $C$ is convex, $\left.\left(\left(y_{n}-n \phi\right)+n \phi\right)\right) /(n+1)=y_{n} /(n+1)$ is in $C$. Since $l\left(y_{n}\right)=l\left(x_{n}\right)+n l(\phi)=0$, $y_{n} /(n+1)$ is in $C \cap Y$. But, since $C$ is bounded, $\left(y_{n}-n \phi\right) / n \rightarrow 0$; that is, $y_{n} / n$ $-\phi \rightarrow 0$. Furthermore $y_{n} /(n+1)-y_{n} / n=-y_{n} / n(n+1)=-(1 / n)\left(y_{n} /(n+1)\right)$ $\rightarrow 0$. Hence $y_{n} /(n+1) \rightarrow \phi$.

Proof of the theorem. It is clear that there is no loss in generality in dealing only with subspaces of $L$ of deficiency one. Let $M$ be such a subspace. Let $l$ be any element of $L-M$ and let $Y$ be the null space of $l$. Obviously $Y_{M}$ and $Y_{L}$ are isomorphic. Hence, by Theorem V-2, $Y_{M}$ has the first countability property. Let $\phi$ be a fixed element of $X-Y$. Let $A_{1}, A_{2}, \cdots$ be a sequence of bounded subsets of $X_{M}$. For each $i=1,2, \ldots$ choose a real number $\mu_{i}$ and a subset $B_{i}$ of $Y$ as follows. If $l$ is bounded on $A_{i}$ let $\mu_{i}$ be such that $|l(x)| \leqq \mu_{i}-1$ for all $x$ in $A_{i}$ and let $B_{i}$ be the set of all $x$ in $Y$ such that $\lambda$ exists with $x+\lambda \phi \in \tilde{C} o\left(A_{i}\right)$. If $l$ is unbounded on $A_{i}$ let $B_{i}=\tilde{C} o\left(A_{i}\right) \cap Y$ and let $\mu_{i}=1$. Then each $B_{i}$ is a bounded subset of $Y_{M}$. Furthermore in the first case $A_{i} / \mu_{i} \subseteq B_{i}+B$ where $B$ is the set of all $\lambda \phi$ with $|\lambda l(\phi)| \leqq 1$ and in the second case by our lemma, $A_{i} / \mu_{i}=A_{i} \subseteq\left(B_{i}\right)^{(1)}$. Now since $Y_{M}$ has the first countability property there exists a sequence $\nu_{1}, \nu_{2}, \cdots$ of positive real numbers such that $\left(B_{1} / \nu_{1}\right) \cup\left(B_{2} / \nu_{2}\right) \cup \cdots$ is $M$ bounded. Since we may suppose that each $\nu_{i}>1$, it follows that $\left(B_{i}+B\right)^{(1)} / \nu_{1} \cup\left(B_{2}+B\right)^{(1)} / \nu_{2} \cup \cdots$ is $M$ bounded. The inclusions concerning the $A$ 's and $B$ 's then tell us that $\left(A_{1} / \nu_{1} \mu_{1}\right) \cup\left(A_{2} / \nu_{2} \mu_{2}\right)$ $\cup \ldots$ is $M$ bounded. It follows then that $X_{M}$ has the first countability property.

The fact that Theorem V-12 does not generalize to arbitrary subsets of $L$ and Theorem V-2 together tell us that the conjugate of a linear system with the first countability property need not have this property. We do not know whether or not the first countability property is invariant under passage to an arbitrary quotient system. 
We turn now to some further considerations involving bounded closure.

Theorem V-13. Let $X$ be a linear space and let $L_{1} \subseteq L_{2} \subseteq L_{3} \subseteq \cdots$ be an ascending sequence of subspaces of $\left(X^{*}\right)_{x}$ each of which is boundedly closed and has the first countability property. Then $L=L_{1}+L_{2}+\cdots$ is boundedly closed.

Proof. Let $l$ be any member of $X^{*}-L$. For each $i=1,2, \cdots$ let $B_{i}$ be an $L_{i}$ bounded subset of $X$ on which $l$ is unbounded. Then for each $i, B_{i}, B_{i+1}, \cdots$ are all $L_{i}$ bounded. Hence there exist positive real numbers $\gamma_{i}^{i}, \gamma_{i+1}^{i}, \cdots$ such that $\left(B_{i} / \gamma_{i}^{i}\right) \cup\left(B_{i+1} / \gamma_{i+1}^{i}\right) \cup \cdots$ is $L_{i}$ bounded. Let $\gamma_{i}$ be the largest of the numbers $\gamma_{i}^{1}, \gamma_{i}^{2}, \cdots, \gamma_{i}^{i}$. Then $\gamma_{j}^{i} \leqq \gamma_{j}$ whenever $\gamma_{j}^{i}$ is defined. Hence if we let $A_{i}=B_{i} / \gamma_{i}, A_{i} \cup A_{i+1} \cup \cdots$ will be $L_{i}$ bounded for each $i=1,2, \cdots$. Let $x_{i}$ be an element of $A_{i}$ such that $\left|l\left(x_{i}\right)\right|>i$. Then it is clear that $\left(x_{1}, x_{2}, \cdots\right)=\left(x_{1}, x_{2}, \cdots, x_{i-1}\right) \cup\left(x_{i}, x_{i+1}, \cdots\right)$ is $L_{i}$ bounded for each $i=1,2, \cdots$ and hence is $L$ bounded. On the other hand, $l\left(x_{1}\right), l\left(x_{2}\right), \cdots$ is not $L$ bounded. Thus $L$ is boundedly closed.

Corollary 1. If $X$ is a linear space and $L$ is an $\aleph_{0}$-dimensional subspace of $\left(X^{*}\right)_{X}$ then $L$ is boundedly closed.

CoRollary 2. If $X$ is a linear space and $L$ and $M$ are boundedly closed subspaces of $\left(X^{*}\right)_{X}$ such that $L$ has the first countability property and $M$ is $\aleph_{0}$-dimensional, then $L+, M$ is boundedly closed.

We close this section with an example, suggested to the author by P. Erdös, showing that the first countability requirement in the statement of Theorem V-13 cannot be omitted.

Let $X$ be an arbitrary infinite-dimensional linear space. Let $\left\{\phi_{i}^{j}\right\}$, $i, j=1,2, \cdots$, be a matrix of countably many linearly independent members of $X$. Let $\left\{\psi_{\alpha}\right\}$ be a set of linearly independent elements of $X$ such that the $\phi_{i}^{j}$ and the $\psi_{\alpha}$ together form a Hamel basis $H$ for $X$. Let $L$ be the set of all members $l$ of $X^{*}$ which are bounded on $H$. For each $i=1,2, \cdots$, let $L_{i}$ be the set of all $l$ in $L$ such that $l\left(\phi_{i}^{1}\right), 2 l\left(\phi_{i}^{2}\right), 3 l\left(\phi_{i}^{3}\right), \cdots$ is a bounded sequence of real numbers. For each $j=1,2, \cdots$ let $M_{j}=L_{j} \cap L_{j+1} \cap L_{j+2} \cap \ldots$. Since, as is readily verified, the set of all linear functionals bounded on a fixed set is boundedly closed it follows that $L$ and all of the $L_{i}$ and $M_{j}$ are boundedly closed. Furthermore it is obvious that $M_{1} \subseteq M_{2} \subseteq M_{3} \subseteq \ldots$. We shall show that while $M=M_{1}+M_{2}+M_{3} \dot{+} \ldots$ is properly contained in $L$, the bounded closure of $M$ is $L$ and hence that $M$ is not boundedly closed.

That the inclusion $M \subseteq L$ is proper is immediate. The functional which is one on all elements of $H$ is obviously in $L$ and not in $M$. We turn now to the proof that $L=\bar{M}$. Suppose if possible that $x_{1}, x_{2}, \ldots$ is a sequence of elements of $X$ which is $M$ bounded but not $L$ bounded. For each $n=1,2, \cdots$, $x_{n}=y_{n}+\sum_{i, j n} c_{i}^{j} \phi_{i}^{j}$ where $y_{n}$ is a finite linear combination of the $\psi_{\alpha}$ and all but a finite number of the ${ }_{n} c_{l}^{s}$ (for fixed $n$ ) are zero. It follows at once from the 
definition of $M$ that $y_{1}, y_{2}, \cdots$ is $L$ bounded and that for each $i, \sum_{j n} c_{i}^{\prime} \phi_{i}^{j}$ is $L$ bounded as a sequence in $n$. Thus for each $k=1,2, \sum_{i=k, k+1}, \ldots ; j=1,2, \ldots{ }_{n} c_{i}^{j} \phi_{i}^{j}$ is not $L$ bounded. For each $x$ in $X$ let $\|x\|$ be the sum of the absolute values of the coefficients obtained when $x$ is expressed as a finite linear combination of the members of $H$. It is clear that \|\| is a norm whose norm set is $L$. For each $k=1,2, \cdots$ and $n=1,2, \cdots$ let $\mu(n, k)=\sum_{i=k, k+1}, \ldots, j=1,2, \ldots\left|{ }_{n} c_{i}^{j}\right|$ and let $\nu(n, k)=\sum_{i=1,2, \cdots, k-1 ; j=1,2, \cdots}\left|{ }_{n} c_{i}^{j}\right|$. Then it follows from our remarks that $\mu(n, k)$ is unbounded in $n$ for each $k$ and that $\nu(n, k)$ is bounded in $n$ for each $k$. Let $\nu(k)=1$.u.b. ${ }_{n=1,2}, \ldots \nu(n, k)$. By first choosing $k_{1}$ then $n_{1}$ then $k_{2}$, and so on, it is clear that we may choose two sequences of positive integers $k_{1}<k_{2}<k_{3}<\cdots$ and $n_{1}<n_{2}<n_{3}<\cdots$ such that $\mu\left(n_{1}, k_{1}\right)>1+\nu\left(k_{1}\right)$, $\mu\left(n_{1}, k_{2}\right)=0$ and for each $s=2,3, \cdots, \mu\left(n_{s}, k_{s}\right)>s+\nu\left(k_{s}\right)$ and $\mu\left(n_{s}, k_{s+1}\right)=0$. Now define a matrix $\left\{\gamma_{i}^{j}\right\}, i, j=1,2, \cdots$, as follows. For $i<k_{1}, \gamma_{i}^{j}=0$. For $k_{s} \leqq i<k_{s+1}, \gamma_{i}^{j}$ is 1,0 , or -1 according as ${ }_{n_{s}} c_{t}^{j}$ is positive, zero, or negative. Let $l$ be the unique linear functional on $X$ such that $l\left(\phi_{i}^{j}\right)=\gamma_{i}^{j}, i, j=1,2, \cdots$, and $l\left(\psi_{\alpha}\right)=0$ for all $\alpha$. Then since $\left\{l\left(\phi_{l}^{j}\right)\right\}$ is bounded and for each $i$ all but a finite number of the $l\left(\phi_{i}^{j}\right)$ are zero it follows that $l$ is in $M$. On the other hand a simple calculation shows us that $\left|l\left(x_{n_{\ell}}\right)\right|>s$ for $s=1,2, \cdots$. Hence $x_{1}, x_{2}, \cdots$ is not $M$ bounded and we have a contradiction.

It is readily verified that each $L_{i}$ in the above example is a norm set. Hence we have proved the following theorem.

Theorem V-14. Let $X$ be an arbitrary infinite dimensional linear space. Then there exists an ascending sequence of boundedly closed subspaces of $\left(X^{*}\right)_{X}$, $M_{1} \subseteq M_{2} \subseteq M_{3} \subseteq \cdots$, such that $M=M_{1}+M_{2} \dot{+} \cdots$ is not boundedly closed. This is the case even if we demand that each $M_{i}$ be not only relatively bounded but the intersection of countably many norm sets.

COROLLARY. The intersection of countably many norm sets need not have the first countability property.

6. The second countability property. As we shall see later and as the reader can probably verify for himself now, an $\boldsymbol{\aleph}_{0}$-dimensional subspace of an $\left(X^{*}\right)_{X}$ cannot be simple. Hence since it has the first countability property it cannot have the second. In other words, we cannot expect an analogue of Theorem $\mathrm{V}-11$ to hold for the second countability property. We do have, however, the following theorem.

TheOREM V-15. Let $X$ be a linear space and let $L$ and $M$ be subspaces of $\left(X^{*}\right)_{X}$ which have the second countability property. Then $L+M$ also has the second countability property.

Proof. Let $B_{1}, B_{2}, \cdots$ be a basis $\left({ }^{24}\right)$ for the $L$ bounded sets and let

(24) That is, a family of bounded sets whose subsets constitute all bounded sets. 
$C_{1}, C_{2}, \cdots$ be a basis for the $M$ bounded sets. Then the $C_{i} \cap B_{j}, i, j=1,2, \cdots$, obviously constitute a basis for the $L \dot{+} M$ bounded sets. Hence $L \dot{+} M$ has the second countability property.

With respect to intersections the situation is a little better than in the case of the first countability property. We shall show in the last chapter that a relatively bounded and boundedly closed subspace of an $\left(X^{*}\right)_{X}$ has the second countability property if and only if it is an intersection of countably many norm sets and hence that for relatively bounded( $\left.{ }^{25}\right)$ and boundedly closed subspaces the second countability property is preserved under countable intersections. Whether or not the second countability property is preserved under general countable intersections is not known.

The analogue of Theorem V-12 is true.

TheOREM V-16. Let $X$ be a linear space and let $L$ be a subspace of $\left(X^{*}\right)_{X}$ having the second countability property. Then every subspace of $L$ having finite deficiency in $L$ also has the second countability property.

Proof. Just as in Theorem V-12, it is sufficient to prove that whenever $Y$ has deficiency one in $X$ and $M$ is a subspace of $X^{*}$ such that $Y_{M}$ has the second countability property then $X_{M}$ has the second countability property. Let $A_{1}, A_{2}, \cdots$ be a basis for the bounded sets in $Y_{M}$. Let $\phi$ be a fixed element of $X-Y$ and for each $n=1,2, \cdots$ let $B_{n}=A_{n} \cup$ (set of all $\lambda \phi$ with $|\lambda| \leqq n)$. Finally for each $n=1,2, \cdots$ let $C_{n}=\left(\tilde{C} o\left(B_{n}\right)\right)^{(1)}$. Then each $C_{n}$ is bounded and has the property that $\tilde{C} o\left(C_{n}\right)=C_{n}$. We shall show that $C_{1}, C_{2}, \cdots$ is a basis for the bounded subsets of $X_{M}$. Let $l$ be the linear functional such that $l(\phi)=1$ and $l(y)=0$ for all $y$ in $Y$. Let $D$ be any bounded subset of $X_{M}$. If $l$ is unbounded on $D$ then, by the lemma connected with Theorem V-12, $\tilde{C} o(D) \cap Y$ is sequentially dense in $\tilde{C} o(D)$. But $\tilde{C} o(D) \cap Y \subseteq A_{n} \subseteq B_{n}$ for some $n=1,2, \cdots$. Thus $(\tilde{C} o(D) \cap Y)^{(1)} \subseteq\left(\tilde{C} o\left(B_{n}\right)\right)^{(1)}=C_{n}$. Hence $D \subseteq \tilde{C} o$, $(D) \subseteq C_{n}$. If, on the other hand, $l$ is bounded on $D$ it follows that the set of all $x-l(x) \phi$ with $x$ in $2 D$ and the set of all $l(x) \phi$ with $x$ in $2 D$ are both bounded. Thus for some $m$ and $n=1,2, \cdots, 2 D \subseteq B_{n}+B_{m}$. It follows at once that $D \subseteq C o\left(B_{\tilde{n}}\right)$ where $B_{\bar{n}}$ contains both $B_{n}$ and $B_{m}$. In any case then $D$ is in some $C_{n}$ and $C_{1}, C_{2}, \ldots$ must be a basis for bounded sets. This completes the proof of the theorem.

Like the first countability property the second countability property is not in general preserved under passage to a subspace or to the conjugate system. That the analogue of Theorem V-13 for the second countability property is not true follows from Theorem V-14 and the remarks following Theorem $\mathrm{V}-15$.

7. Simplicity. Let $X$ be a linear space and let $L$ be a simple subspace of $\left(X^{*}\right)_{X}$. Let $B$ be a bounded subset of $X_{L}$ such that the sets $B, 2 B, 3 B, \cdots$

(25) As we shall see, an $L$ is relatively bounded if and only if it is contained in a norm set. 
form a basis for the bounded sets. It is clear that we may always choose $B$ so as to be convex, contain $-x$ with $x$, and so that its intersection with every one-dimensional subspace of $X$ is a closed subset. Accordingly by a well known procedure we may define a function \|\| on $X$ to the non-negative reals which has all the properties of a norm except that $\|x\|$ may be zero when $x$ is not zero and which is such that $x$ is in $B$ if and only if $\|x\| \leqq 1$. We call such a function a pseudo-norm $\left({ }^{26}\right)$ and define the pseudo-norm set of a pseudo-norm \|\| to be the set of all $l$ in $X^{*}$ such that whenever $\left\|x_{1}\right\|,\left\|x_{2}\right\|, \ldots$ is bounded then $l\left(x_{1}\right), l\left(x_{2}\right), \cdots$ is also bounded. It is readily verified that the pseudo-norm set of a pseudo-norm which happens to be a norm is a norm set and that a pseudo-norm set is a norm set if and only if it is total. The following theorem is an obvious consequence of the foregoing remarks.

TheOREM V-17. Let $X$ be a linear space and let $L$ be a subspace of $X^{*}$. Then $L$ is simple if and only if its bounded closure is a pseudo-norm set and if $L$ is total it is simple if and only if its bounded closure is a norm set.

As we shall devote the whole of Chapter VII to the study of norm sets and pseudo-norm sets we shall not study simplicity further here. Many properties of simple $L$ 's are deducible at once from the theorems of $\$ \S 5$ and 6 and the fact that an $L$ is simple if and only if it has both countability properties.

\section{Chapter VI. Uniform Boundedness}

1. Fundamental definitions. Let $X_{L}$ be a linear system and let $A$ be a bounded subset of $X_{L}$. For each $l$ in $L$, l.u.b. ${ }_{x \in A}|l(x)|$ is finite but it is clear that this bound does not exist uniformly for all $l$ in $L$. On the other hand it is conceivable that it exist uniformly for all $l$ in each bounded subset of $X_{L}^{\circ}$; in other words that for each $X$ bounded subset $B$ of $L$ the set of real numbers of the form $l(x)$ where $x$ is in $A$ and $l$ is in $B$ is bounded. However, as we shall see below, even this is true only in certain instances. Thus we are led to make the following definitions. A uniformly bounded subset of a linear system $X_{L}$ is a subset $A$ such that whenever $B$ is a bounded subset of $X_{L}^{\circ}$ then l.u.b. $l \in B$ (l.u.b. $x \in A|l(x)|$ ) is finite. $X_{L}$ is $u$ inform if and only if every bounded subset of $X_{L}$ is uniformly bounded. It is clear that $X_{L}$ is uniform if and only if $X_{L}^{\circ}$ is uniform. For each subset $B$ of $L$ and each $x$ in $X$ let $\|x\|_{B}$ $=$ l.u.b. $l \in B|l(x)| .\|x\|_{B}$ is finite for all $x$ in $X$ if and only if $B$ is a bounded subset of $X_{L}^{\circ}$ and whenever this is the case \|\|$_{B}$ is a pseudo-norm. For each bounded subset $B$ of $X_{L}^{\circ}$ let $L_{B}$ be the pseudo-norm set of \|\|$_{B}$. Then it is clear that a subset $A$ of $X_{L}$ is uniformly bounded if and only if it is $L_{B}$ bounded for each bounded subset $B$ of $X_{L}$. Hence if we call the linear union of all the $L_{B}$ the reach $L_{r}$ of $L$ we may state the following theorem.

${ }^{(26)}$ Not to be confused with the pseudo-norm of Hyers [9]. Cf., however, [26] where the term is used in our sense. 
TheOREM VI-1. Let $X_{L}$ be a linear system. Then the uniformly bounded subsets of $X_{L}$ are identical with the bounded subsets of the reach $X_{L_{r}}$ of $X_{L}$.

It is obvious that for any subspace $L$ of an $\left(X^{*}\right)_{X}, L_{r} \supseteq L$. We do not know whether or not $\left(L_{r}\right)_{r}$ can properly contain $L_{r}$ nor whether $L_{r}$ can fail to be boundedly closed.

\section{Uniformity and simplicity.}

THEOREM VI-2. Every relatively bounded uniform linear system is simple.

Proof. Let $X_{L}$ be relatively bounded and uniform. Since $X_{L}^{\circ}$ is relatively bounded, there exists a bounded subset $B$ of $X_{L}^{\circ}$ whose linear span is $L$. Clearly $L \subseteq L_{B} \subseteq L_{r}$ and hence $\bar{L} \subseteq \bar{L}_{B} \subseteq \bar{L}_{r}$. But since $X_{L}$ is uniform, $\bar{L}=\bar{L}_{r}$. Thus $\bar{L}=\bar{L}_{B}=L_{B}$ and $L$ is pseudo-norm set. It follows from Theorem V-17 that $X_{L}$ is simple.

In order to see that the converse of this theorem is not true we have only to consider a linear system $X_{L}$ in which $X$ is $\aleph_{0}$-dimensional and $L$ is a norm set. If $X_{L}$ were uniform $X_{L}^{\circ}$ would have to be uniform and hence simple. Since $X$ is $\boldsymbol{\aleph}_{0}$-dimensional $X_{L}^{\circ}$ is boundedly closed. Hence $X$ would have to be a norm set as a subspace of $\left(L^{*}\right)_{L}$. However, since any norm set is complete in its natural norm it follows from Theorem I-1 that no norm set can be $\aleph_{0}$-dimensional. Thus $X_{L}$ is simple but not uniform. We do have, however, the following theorem.

THEOREM VI-3. A linear system $X_{L}$ is simple if and only if the reach of its conjugate system is relatively bounded.

Proof. If $X_{L}$ is simple let \|\| be a pseudo-norm defining the bounded sets in $X_{L}$ and let $A$ be the set of all elements in $L$ for which 1.u.b.||x||-1 $|l(x)| \leqq 1$. Clearly $A$ is a uniformly bounded subset of $X_{L}^{\circ}$ and has $L$ for its linear span. Hence the reach of $X_{L}^{\circ}$ is relatively bounded. Conversely, if the reach of $X_{L}^{\circ}$ is relatively bounded there exists a uniformly bounded subset $A$ of $X_{L}^{\circ}$ whose linear span is $L$. Thus $L_{A} \supseteq L$. On the other hand, since $A$ is uniformly bounded, every $L$ bounded subset of $X$ is $L_{A}$ bounded so that $L_{A} \subseteq \bar{L}$. Hence $L \subseteq L_{A} \subseteq \bar{L}$, and since $L_{A}$ is boundedly closed it follows that $L_{A}=\bar{L}$, so that $\bar{L}$ is a pseudo-norm set and $X_{L}$ is simple.

\section{Uniformity and completeness.}

THEOREM VI-4. Every complete linear system is uniform.

Proof. Let $X_{L}$ be a complete linear system, let $x_{1}, x_{2}, \ldots$ be an arbitrary bounded sequence of elements of $X_{L}$ and let $l_{1}, l_{2}, \ldots$ be an arbitrary bounded sequence of elements of $X_{L}^{\circ}$. It will clearly suffice to show that 1.u.b. $i=1,2, \ldots$ (l.u.b. ${ }_{j=1,2}, \ldots\left|a_{i j}\right|$ ) is finite where for $i, j=1,2, \cdots, a_{i j}=l_{i}\left(x_{j}\right)$. Consider the Banach space $(l)$ of all absolutely convergent series of real numbers. Since l.u.b. ${ }_{j=1,2}, \ldots\left|a_{i j}\right|$ is finite for $i=1,2, \cdots$, it follows $[2$, p. 67] 
that $a_{i j}$ as a sequence in $j$ defines a continuous linear functional $m_{i}$ on (l) such that $\left\|m_{i}\right\|=1$. u.b. ${ }_{j=1,2}, \ldots\left|a_{i j}\right|$. Thus in order to prove our theorem we need only show that l.u.b. ${ }_{i=1,2}, \ldots .\left\|m_{i}\right\|$ is finite, and to do this it is enough $\left({ }^{27}\right)$ to prove that $m_{1}(s), m_{2}(s), \cdots$ is bounded for each $s$ in $(l)$. Let $s=s_{1}, s_{2}, \cdots$ be an arbitrary member of $(l)$. For each $n=1,2, \cdots$ let $y_{n}=s_{1} x_{1}+s_{2} x_{2}+\cdots$ $+s_{n} x_{n}$. Then it is readily verified that $y_{1}, y_{2}, \cdots$ is a Cauchy sequence and hence since $X_{L}$ is complete that $y_{n} \rightarrow \bar{x}$ where $\bar{x}$ is in $X$. In particular, for each $i=1,2, \cdots, l_{i}\left(y_{n}\right) \rightarrow l_{i}(\bar{x})$; that is, $m_{i}(s)=\sum_{j=1}^{\infty} s_{j} a_{i j}=l_{i}(\bar{x})$. But since $l_{1}, l_{2}, \ldots$ is a bounded subset of $X_{L}^{\circ}, l_{1}(\bar{x}), l_{2}(\bar{x}), \ldots$ is bounded. Hence $m_{1}(s), m_{2}(s), \cdots$ is bounded and the theorem is proved.

COROLlaRy. Every complete relatively bounded linear system is simple.

Lemma. Let $X$ be a linear space and let $L$ be a boundedly closed subspace of $\left(X^{*}\right)_{X}$. Then $L$ is uniform if and only if $L$ is "sequentially weakly closed" in the sense that whenever $l_{1}, l_{2}, \ldots$ is a sequence of elements of $L$ such that $l_{1}(x), l_{2}(x), \cdots$ converges for all $x$ in $L$ then the function $f$ such that $f(x)$ $=\lim _{n \rightarrow \infty} l_{n}(x)$ is in $L$.

Proof. If $L$ is sequentially weakly closed it is obvious that $X_{L}^{\circ}$ is complete and hence, by Theorem VI-4, uniform. Thus $X_{L}$ is uniform. Conversely suppose that $X_{L}$ is uniform and let $l_{1}, l_{2}, \cdots$ be a sequence of members of $L$ such that $l_{n}(x) \rightarrow f(x)$ for all $x$ in $X$. Obviously $f$ is in $X^{*}$. Suppose that $x_{1}, x_{2}, \cdots$ is a bounded sequence of elements of $X_{L}$. For each $n=1,2, \cdots$, $f\left(x_{n}\right)=\lim _{m \rightarrow \infty} l_{m}\left(x_{n}\right)$ and, since $l_{1}, l_{2}, \cdots$ is a bounded subset of $X_{L}^{\circ},\left\{l_{m}\left(x_{n}\right)\right\}$ is bounded as $m$ and $n$ vary independently over the positive integers. It follows that $f\left(x_{1}\right), f\left(x_{2}\right), \cdots$ is bounded. Thus $f \in \bar{L}=L$ and $L$ is sequentially weakly closed.

The following two theorems are immediate consequences of this lemma.

THEOREM VI-5. A boundedly closed linear system is uniform if and only if its conjugate is complete.

THEOREM VI-6. If $\left\{L_{\alpha}\right\}$ is an arbitrary family of uniform boundedly closed subspaces of an $\left(X^{*}\right)_{X}$ then $\prod L_{\alpha}$ is uniform and boundedly closed.

We shall show later that uniformity differs from the other properties of linear systems that we have studied in that the bounded closure of a uniform linear system need not be uniform.

\section{Stability of uniformity.}

Lemma 1. Let $X_{L}$ be a uniform linear system and let libe a member of $X^{*}-L$. Then $X_{(L+l)}$ is also a uniform linear system.

Proof. Let $m_{1}, m_{2}, \cdots$ be an arbitrary bounded sequence of elements of

${ }^{(27)}$ Because of Théorème 5 on page 80 of [2]. 
$X_{\left(L+\dot{+}_{l}\right)}^{\circ}$ and let $x_{1}, x_{2}, \cdots$ be an arbitrary bounded sequence of elements of $X_{(L+i)}$. We shall show that $\left\{m_{i}\left(x_{j}\right)\right\}$ is bounded as $i$ and $j$ vary independently over the positive integers. For each $i=1,2, \cdots, m_{i}=l_{i}+\lambda_{i} l$ where each $l_{i}$ is in $L$. If $\lambda_{1}, \lambda_{2}, \ldots$ is a bounded sequence of real numbers the boundedness of $\left\{m_{i}\left(x_{j}\right)\right\}$ is obvious. Hence there is no loss in generality in supposing that no $\lambda_{i}$ is zero and that some subsequence $\lambda_{n_{1}}, \lambda_{n_{2}}, \ldots$ tends to infinity. For each $i=1,2, \cdots$ let $f_{i}=l_{i} /\left(-\lambda_{i}\right)$ and let $\nu_{i}=1 /\left(-\lambda_{i}\right)$ so that $m_{i}=\left(f_{i}-l\right) / \nu_{i}$. Since $m_{1}, m_{2}, \cdots$ is bounded there exists a positive function $F$ defined on $X$ such that for all $x$ in $X$ and $i=1,2, \cdots,\left|f_{i}(x)-l(x)\right| \leqq \nu_{i} F(x)$. It follows that for all $x$ in $X$ and $i, k=1,2, \cdots,\left|f_{i}(x)-f_{k}(x)\right| /\left(\nu_{i}+\nu_{k}\right) \leqq F(x)$. Thus since $\left(f_{i}-f_{k}\right) /\left(\nu_{i}+\nu_{k}\right)$ is in $L$ and $X_{L}$ is uniform, there exists a positive real number $K$ such that $\left|f_{i}\left(x_{j}\right)-f_{k}\left(x_{j}\right)\right| /\left(\nu_{i}+\nu_{k}\right) \leqq K$ for all $i, j, k=1,2, \cdots$. But $\nu_{n_{1}}, \nu_{n_{2}}, \cdots$ converges to zero. Hence, since $\left|f_{i}(x)-l(x)\right| \leqq \nu_{i} F(x)$, $f_{n_{1}}(x), f_{n_{2}}(x), \cdots$ converges to $l(x)$. Thus letting $k$ take on the values $n_{1}, n_{2}, \cdots$ and passing to the limit we conclude that $\left|f_{i}\left(x_{j}\right)-l\left(x_{j}\right)\right| / \nu_{i} \leqq K$, for $i, j=1,2, \cdots$. But since $\left(f_{i}\left(x_{j}\right)-l\left(x_{j}\right)\right) / \nu_{i}=m_{i}\left(x_{j}\right)$, this completes the proof of the lemma.

Lemma 2. Let $X_{L}$ be a uniform linear system and let $Y$ be a subspace of $X$ having deficiency one in $X$. Then $Y_{L}$ is also a uniform linear system.

Proof. Let $l$ be a member of $X^{*}$ whose null space is $Y$. By Lemma 1 , $X_{(L \dot{+} \imath)}$ is uniform. But it is clear that $X_{(L \dot{+} \imath)}$ is isomorphic to the direct union of $Y_{L}$ with a one-dimensional linear system. Furthermore it is an obvious consequence of the definitions concerned that the direct union of two linear systems is uniform if and only if each factor is uniform. Thus $Y_{L}$ is uniform.

Theorem VI-7. Let $X$ be a linear space, let $Y$ be a subspace of $X$ of finite deficiency and let $L$ and $M$ be subspaces of $X^{*}$ such that $M$ is a subspace of $L$ having finite deficiency in $L$. Then if any one of the linear systems $X_{L}, Y_{L}$, $X_{M}, Y_{M}$ is uniform, so are all the rest.

Proof. The truth of this theorem is an obvious consequence of Lemmas 1 and 2 and the fact that a linear system is uniform if and only if its conjugate is uniform.

5. Applications to the theory of normed linear spaces. Let $X_{L}$ be the linear system of a normed linear space and let $B$ be the set of all $x$ in $X$ with $\|x\| \leqq 1$. Then it is clear that $A \subseteq L$ is uniformly $X$ bounded if and only if l.u.b. $x_{x \in B}|l(x)|$ is bounded for $l$ in $A$; that is, if and only if $A$ is norm bounded. Thus the norm bounded sets in $L$ are precisely the uniformly bounded subsets of $X_{L}^{\circ}$, and $X_{L}$ is uniform if and only if the norm bounded subsets of $L$ are precisely the bounded subsets of $X_{L}^{\circ}$. It follows from this last remark that if $X$ is a normed linear space with a uniform linear system and $Y$ is a normed linear space containing $X$ and contained in its completion, then $Y$ also has a uniform linear system. Since the linear system of any complete normed linear 
space is uniform, this tells us that a normed linear space will have a uniform linear system if and only if it is "sufficiently near to its completion." Accordingly we call a normed linear space whose linear system is uniform an almost complete normed linear space. Theorem VI-7 tells us that any normed linear space with finite deficiency in its completion is almost complete. The proof of The eròme 5 on page 80 of [2] tells us that every normed linear space of the second category is almost complete. An example of Hausdorff $[8$, p. 303] shows that not every normed linear space of the second category has finite deficiency in its completion. We do not know however whether or not an almost complete normed linear space is necessarily of the second category. In any case there is an important theorem of Banach( $\left.{ }^{28}\right)$ which can be strengthened by replacing the hypothesis that a certain space be of the second category by the hypothesis that it be almost complete.

THEOREM VI-8. Let $X$ be a linear space and let \|\| and \|\|$_{1}$ be two norms in $X$ such that under \|\|$X$ is complete, under \|\|$_{1} X$ is almost complete, and for all $x$ in $X,\|x\|_{1} \leqq\|x\|$. Then the norms \|\| and \|\|$_{1}$ are equivalent.

Proof. Let $U$ be the identical mapping from $X$ under \|\| into the completion of $X$ under \|\|$_{1}$. Then $U$ is a continuous linear operator. Let $L$ and $L_{1}$ be the norm sets of \|\| and \|\|$_{1}$ respectively. Since $L$ and $L_{1}$ are both uniform, $L_{1}$ has the same bounded sets as norm set as it does as a subset of the norm set $L$. Hence the adjoint $\bar{U}$ of $U$ is an isomorphism between $L_{1}$ as a norm set and $L_{1}$ as a subspace of the norm set $L$. Thus the hypotheses of Theorème 1 on page 146 of [2] are satisfied and we conclude that $U$ maps $X$ onto the whole of the completion of $X$ under \|\|$_{1}$; in other words $X$ is complete under \|\|$_{1}$. It now follows from Théorème 6 on page 41 of [2] that \|\| and \|\|$_{1}$ are equivalent.

We conclude the chapter by using Theorem VI-8 to construct the example of a uniform linear system with a nonuniform bounded closure promised earlier. Let $X$ be a nonreflexive Banach space, let $L$ be the norm set of $X$ and let $Z$ be the norm set of $L, X \subseteq Z \subseteq L^{*}$. Suppose that it is possible to find a closed subspace $Y$ of $Z$ such that $Y \cap X=0$ and $Y \dot{+} X$ is not closed. For each element $y+x$ in $Y+X$ where $y$ is in $Y$ and $x$ is in $X$ let $\|y+x\| \|$ $=\|y\|+\|x\|$. It is then clear that $\|z\| \leqq\|z\| \|$ for all $z$ in $Y \dot{+} X$, that $Y \dot{+} X$ is complete under $|\|\|| \|$ and that \|\| and $\|\mid\|$ are not equivalent. Hence it follows from Theorem VI-8 that $Y+X$ under \|\| is not almost complete; in other words that the bounded closure of the linear system $(Y \dot{+} X)_{L}$ is not uniform. On the other hand, since $X_{L}$ and $Z_{L}$ are uniform it follows that $(Y+X)_{L}$ is uniform. Thus to complete the construction of our example we have only to prove the existence of a nonreflexive Banach space whose second conjugate has a subspace of the sort described. This is easily done as follows.

(28) The specialization to normed linear spaces of Thérème 6 on page 41 of [2]. 
Let $X$ be the subspace of the Banach space (c) [2, p. 6] consisting of the convergent to zero sequences. Then as is well known $Z$ is the Banach space $(m)$. Divide the positive integers into $\aleph_{0}$ classes $I_{1}, I_{2}, \ldots$ each containing $\aleph_{0}$ members and for each $n=1,2, \cdots$, let $s^{n}$ be the sequence $s_{1}^{n}, s_{2}^{n}, \ldots$ such that $s_{j}^{n}=1$ if $j$ is in $I_{n}$ and is otherwise zero. Let $e^{n}$ be the sequence $e_{1}^{n}, e_{2}^{n}, \cdots$ such that $e_{j}^{n}=\delta_{j}^{n}$. Finally let $Y$ be the closure of the linear span of the sequences $e^{1}+s^{1}, e^{2}+s^{2} / 2, e^{3}+s^{3} / 3, \ldots$. It is readily verified that $Y \cap X=0$. On the other hand, since $\left\|e^{n}+s^{n} / n\right\| \geqq\left\|e^{n}\right\|=1$ and $\left\|e^{n}+s^{n} / n-e^{n}\right\|$ $=1 / n$ for $n=1,2, \cdots$ it follows that $X$ and $Y$ are not completely disjoint and hence by the theorems of Chapter III that $X \dot{+} Y$ is not closed.

\section{Chapter VII. Norm Sets}

Since pseudo-norm sets appear naturally in dealing with intersections and since most of our theorems are true for pseudo-norm sets as well as for norm sets we shall often state and prove them in terms of the more general concept.

1. Characterizations. Let $X$ be a linear space and let $L$ be a subspace of $\left(X^{*}\right)_{X}$. We wish to study conditions under which $L$ is a pseudo-norm set. We already know of two ways in which $L$ may fail to be a pseudo-norm set. It may be "too big" in that it is not relatively bounded and it may be "too small" in that it is not boundedly closed. However there is a great deal more to being a pseudo-norm set than being not too big and not too small. We shall see below that there are many examples of $L$ 's which are boundedly closed and relatively bounded but are not pseudo-norm sets; as a matter of fact we have one class of examples already - the $\boldsymbol{N}_{0}$-dimensional subspaces of norm sets. On the other hand we shall show that $L$ is a pseudo-norm set if and only if certain related linear systems are relatively bounded and boundedly closed.

TheOREM VII-1. Let $X$ be a linear space and let $L$ be a subspace of $\left(X^{*}\right)_{X}$. Then $L$ is a pseudo-norm set if and only if the completion of the regularization of $X_{L}$ is relatively bounded and boundedly closed.

Proof. If $L$ is a pseudo-norm set then it is clear that the regularization of $X_{L}$ is the linear system of a normed linear space and that its completion is isomorphic to the linear system of the completion of the space. But the linear system of any normed linear space is relatively bounded and boundedly closed. To prove the converse we observe first that $L$ is a pseudo-norm set if and only if the regularization of $X_{L}$ is the linear system of a normed linear space. Thus we need only consider the case in which $X_{L}$ is regular. Now by Theorem VI-4 the completion of $X_{L}$ is uniform. Hence, by Theorem VI-2, since it is relatively bounded it is simple and, by Theorem V-17, since it is boundedly closed it is the linear system of a normed linear space.

As an immediate corollary of Theorems V-17 and VI-3 we may state the following theorem.

THEOREM VII-2. If $X$ is a linear space and $L$ is a subspace of $\left(X^{*}\right)_{X}$ then $L$ 
is a pseudo-norm set if and only if $X_{L}$ is boundedly closed and the reach of $X_{L}^{\circ}$ is relatively bounded.

Our next theorem tells us how $L$ 's that satisfy the two necessary conditions of being relatively bounded and boundedly closed are related to $L$ 's that are actually norm sets.

TheOREM VII-3. Let $X$ be a linear space and let $L$ be a subspace of $\left(X^{*}\right)_{X}$. Then $L$ is relatively bounded and boundedly closed if and only if it is the intersection of the members of a family of norm sets.

Proof. Half of the theorem is obvious in the light of what we know about bounded closure and relative boundedness. To prove the other half suppose that $L$ is relatively bounded and boundedly closed and let $l$ be any member of $X^{*}-L$. We shall show that there is a norm set which contains $L$ and does not contain $\bar{l}$. To do this we first show that there exists an $L$ bounded sequence of linearly independent members of $X, x_{1}, x_{2}, \ldots$ such that $\bar{l}\left(x_{1}\right), \bar{l}\left(x_{2}\right), \ldots$ is not bounded. It follows at once from the bounded closure of $L$ that $z_{1}, z_{2}, \cdots$ exists with the first and third properties. Hence if $z_{1}+z_{2}+\cdots=M$ is infinite-dimensional, a suitable subsequence of $z_{1}, z_{2}, \ldots$ will have all three. Suppose that $M$ is finite-dimensional. Then since, as on $M, \bar{l}$ is not in the bounded closure of $L$ and since in finite-dimensional linear systems closure and bounded closure are equivalent, there must exist $\bar{x}$ in $M$ such that $\bar{l}(\bar{x})=1$ and $l(\bar{x})=0$ for all $l$ in $L$. Since $L+l$ is relatively bounded there exists an $L \dot{+} l$ bounded sequence $y_{1}, y_{2}, \ldots$ of linearly independent $\left({ }^{29}\right)$ members of $X$. For each $n=1,2, \cdots$ let $x_{n}=y_{n}+n \bar{x}$. Since we may clearly suppose that $\bar{x}$ is not in $y_{1}+y_{2}+\cdots$, it is verified at once that $x_{1}, x_{2}, \cdots$ is a linearly independent set, is $L$ bounded, and is such that $\vec{l}\left(x_{1}\right), \vec{l}\left(x_{2}\right), \ldots$ is not bounded. Let $N$ be a complement of $M$. Since $N_{L}$ is relatively bounded there exists an $L$ bounded Hamel basis $\left\{y_{\alpha}\right\}$ for $N$. $\left\{x_{n}\right\} \cup\left\{y_{\alpha}\right\}$ is then a bounded Hamel basis for $X$. For each $x$ in $X$ let $\|x\|=\| c_{1} x_{1}+\cdots+c_{n} x_{n}+d_{1} y_{\alpha_{1}}+\cdots$ $+d_{n} y_{\alpha_{n}}||=\left|c_{1}\right|+\cdots+\left|c_{n}\right|+\left|d_{1}\right|+\cdots+\left|d_{n}\right|$. It is obvious that $L$ is in the norm set of \|\| and that $\bar{l}$ is not.

Corollary. If $X$ is a linear space and $L$ is a subspace of $X^{*}$ then $L$ is relatively bounded if and only if it is contained in a norm set.

We conclude $\$ 1$ with a characterization of a somewhat different nature.

TheOREM VII-4. Let $X$ be a linear space and let $L$ be a subspace of $\left(X^{*}\right)_{X}$. Then $L$ is a pseudo-norm set if and only if $L$ is the linear span of a convex subset $K$ of $X^{*}$ which is bicompact in the weak topology $\left({ }^{30}\right)$ in $X^{*}$ defined by the members of $X$.

${ }^{(29)}$ If $X$ is finite-dimensional the theorem is trivial.

${ }^{(30}$ ) That is, the weakest topology in $X^{*}$ such that all members of $X^{* *}$ defined by members of $X$ are continuous. 
Proof. Half of the theorem is an immediate consequence of the well known fact that the unit sphere of the conjugate of a Banach space is bicompact in the weak topology defined by the elements. The other half is an easy generalization of a theorem of Krein and Smulian [13, Theorem 16]. They prove that the linear span of a "regularly convex" subset of the conjugate of a Banach space is a pseudo-norm set. However, using the methods used by Mazur [20] in establishing a similar theorem for normed linear spaces, it is easy to show that every subset of $X^{*}$ which is convex and closed in the weak topology is regularly convex. Furthermore inspection of the arguments of Krein and Smulian shows that the assumption that $K$ is contained in the norm set of a Banach space is not needed.

2. Linear unions of pseudo-norm sets. We have shown that if $L$ and $M$ are boundedly closed subspaces of an $\left(X^{*}\right)_{X}$ then $L+M$ is boundedly closed provided that $M$ is finite-dimensional or that $M$ is $\boldsymbol{\aleph}_{0}$-dimensional and $L$ has the first countability property. The following corollary of Theorem VII-4 tells us that we can also draw this conclusion if both $L$ and $M$ are simple.

Theorem VII-5. If $X$ is a linear space and $L$ and $M$ are pseudo-norm sets in $\left(X^{*}\right)_{X}$, then $L+M$ is a pseudo-norm set.

Proof. Consider $X^{*}$ in its weak $X$ topology. By Theorem VII-4, $L=K \dot{+}$ and $M=J \dot{+}$ where $K$ and $J$ are convex and bicompact. By a well known theorem $\left({ }^{31}\right)$ in the theory of topological groups $K+J$ is bicompact. Since $K+J$ is obviously convex it follows from Theorem VII-4 that $(K+J)+$ is a pseudo-norm set. But $(K+J) \dot{+}=L \dot{+} M$. Thus $L \dot{+} M$ is a pseudo-norm set.

It follows at once from Theorem VII-5 that the linear union of any finite number of pseudo-norm sets is again a pseudo-norm set. The following theorem tells us that the finiteness condition very definitely cannot be relaxed.

TheOREM VII-6. Let $X$ be a linear space and let $L_{1}, L_{2}, \cdots$ be an arbitrary sequence of pseudo-norm sets in $\left(X^{*}\right)_{X}$. Then $L=L_{1}+L_{2} \dot{+} \cdots$ is a pseudonorm set if and only if for some $n_{0}=1,2, \cdots, L=L_{1} \dot{+} L_{2}+\cdots+L_{n_{0}}$.

Proof. Because of Theorem VII-5 half of the theorem is trivial, and in proving the other half we may assume that $L_{1} \subseteq L_{2} \subseteq L_{3} \subseteq \ldots$. For each $n=1,2, \cdots$ let \|\|$_{n}$ be a pseudo-norm whose pseudo-norm set is $L_{n}$. Because of the relation between $L_{n}$ boundedness and $L_{n+1}$ boundedness it is clear that we may choose these pseudo-norms so that $\|x\|_{n} \leqq\|x\|_{n+1}$ for all $x$ in $X$ and each $n=1,2, \cdots$. Suppose that $L$ is a pseudo-norm set and let \|\| be a corresponding pseudo-norm. If $L \neq L_{n}$ for any $n=1,2, \cdots$ then for each $n$ there is an $L_{n}$ bounded set which is not $L$ bounded. Hence for each $n=1,2, \cdots$ there exists $x_{n}$ in $X$ such that $\left\|x_{n}\right\|_{n}<1$ and $\left\|x_{n}\right\|=n$. Now for each pair of positive integers $m$ and $n$ with $m>n,\left\|x_{m}\right\|_{n} \leqq\left\|x_{m}\right\|_{(n+1)} \leqq \cdots$

(31) See $[27$, p. 16] for a proof. 
$\leqq\left\|x_{m}\right\|_{m}<1$. Hence for $n=1,2, \cdots$ the sequence $\left\|x_{1}\right\|_{n},\left\|x_{2}\right\|_{n}, \cdots$ is bounded. Hence $x_{1}, x_{2}, x_{3}, \cdots$ is $L_{n}$ bounded for all $n$ and consequently $L$ bounded. But this is impossible since $\left\|x_{n}\right\|=n$. Thus for some $n=1,2, \cdots$, $L=\dot{L}_{n}$.

\section{Intersections of pseudo-norm sets.}

TheOREM VII-7. If $X$ is a linear space and $L$ and $M$ are pseudo-norm sets in $\left(X^{*}\right)_{X}$, then $L \cap M$ is a pseudo-norm set.

Proof $\left.{ }^{82}\right)$. Let $K$ and $J$ be convex subsets of $X^{*}$ which are bicompact in the weak $X$ topology and such that $L=K \dot{+}$ and $M=J \dot{+}$. Then $L \cap M$ $=(K \cap J) \dot{+}$. Since $K \cap J$ is bicompact and convex, $L+M$ is a pseudo-norm set.

It follows from this theorem that the intersection of two norm sets is a norm set if and only if it is total. That it need not be total is an immediate consequence of Theorem V-9. As a matter of fact, using Theorem V-9 we can prove the following theorem.

THEOREM VII-8. If $X$ is an infinite-dimensional linear space then every pseudo-norm set in $\left(X^{*}\right)_{X}$ is the intersection of two norm sets.

Proof. Let $M$ be a pseudo-norm set in $\left(X^{*}\right)_{x}$ and let $Y$ be a complement of $M^{\prime}$ in $X$. Then, as on $Y$, any pseudo-norm \|\| associated with $L$ is a norm. Let $\|\mid\|$ be any norm defined on $M^{\prime}$, and for each $z$ in $M^{\prime}$ and each $y$ in $Y$ let $\|z+y|\|=\| z|\|+\|y\|$. It is readily verified that $\||\||$ is a norm defined throughout $X$. Let $L$ be its norm set. It is clear that $M \subseteq L$. Furthermore it is clear that $M^{\prime \prime} \cap L=M$ and hence that $M$ is a closed subspace of $X_{L}^{\circ}$. Thus by Theorem V-9 there exists an automorphism $T^{*}$ of $\left(X^{*}\right)_{X}$ such that $T^{*}(L)$ $\cap L=M$. Since $L$ and $T^{*}(L)$ are norm sets, this completes the proof of the theorem.

Theorem VII-7 tells us that the intersection of any finite number of pseudo-norm sets is again a pseudo-norm set. That the cardinal number restriction cannot be completely removed follows from the first section of this chapter. It follows from the corollary to Theorem V-14 that it cannot even be relaxed. On the other hand the analogue of Theorem VII- 6 is not true and there are many non-trivial cases of infinite intersections of pseudo-norm sets which are themselves pseudo-norm sets. Examples are furnished us by the following two theorems.

THEOREM VII-9. If $X$ is a linear space and $\left\{L_{\alpha}\right\}$ is an arbitrary family of uniform pseudo-norm sets in $\left(X^{*}\right)_{X}$, then $L=\prod L_{a}$ is also a uniform pseudonorm set.

(82) A more elementary but slightly longer proof may be given by starting with pseudonorms associated with $L$ and $M$ and considering the least upper bound of all the pseudo-norms which are less than or equal to both. 
Proof. By Theorem VI-6, $L$ is uniform and boundedly closed. Obviously $L$ is relatively bounded. Hence by Theorem VI-2 it is simple. Since it is simple and boundedly closed, it is a pseudo-norm set.

THEOREM VII-10. If $L$ is a pseudo-norm set and if $\left\{L_{\alpha}\right\}$ is a family of pseudo-norm sets each of which has finite deficiency in $L$, then $\prod L_{\alpha}$ is also a pseudo-norm set.

Proof. We observe first that, since every subspace of $L$ which contains an $L_{\alpha}$ is again a pseudo-norm set, every $L_{\alpha}$ is an intersection of pseudo-norm sets of deficiency one in $L$. Thus we need only consider the case in which each $L_{\alpha}$ has deficiency one in $L$. Furthermore we shall suppose that $L$ is a norm set. That the general case may be reduced to this one follows from an easy argument that we leave to the reader. Let \|\| be a norm in $X$ corresponding to $L$ and let $\tilde{X}$ be the completion of $X$ under \|\| . Since each $L_{\alpha}$ is boundedly closed and of deficiency one, it follows from Theorem IV-9 that it is the null space of a member $\left.{ }^{33}\right)$ of $\tilde{X}$. Thus each $L_{\alpha}$ is a closed subspace of $\tilde{X}_{L}^{\circ}$. Hence $\prod L_{\alpha}$ is a closed subspace of $\tilde{X}_{L}^{\circ}$. But it follows from Theorems V-9 and VII-7 that every closed subspace of $\tilde{X}_{L}^{\circ}$ is a pseudo-norm set in $\left(\tilde{X}^{*}\right)_{\tilde{X}}$. Thus $\prod L_{\alpha}$ is a pseudo-norm set as on $\tilde{X}$ and hence as on $X$.

Although the analogue for intersections of Theorem VII-6 is not true there is an interesting quasi-analogue which is true. We obtain this result from Theorem VII- 6 by means of a certain duality between intersections and linear unions which we shall now proceed to describe.

Let $X$ be a linear space and let $L$ be a total subspace of $X^{*}$. Then $X$ regarded as a subspace of $L^{*}$ is also total. Let $\mathcal{F}_{1}$ be the family of all boundedly closed subspaces of $X^{*}$ which contain $L$ and let $\mathcal{F}_{2}$ be the family of all boundedly closed subspaces of $L^{*}$ which contain $X$. We define an operation $F \rightarrow F^{-}$ which takes each member of $\mathcal{F}_{1}$ into a member of $\mathcal{F}_{2}$ and each member of $\mathcal{F}_{2}$ into a member of $\mathscr{F}_{1}$ as follows. If $F$ is in $\mathscr{F}_{1}\left(\mathscr{F}_{2}\right)$ let $\mathcal{B}_{F}$ be the family of all subsets of $L(X)$ which are uniformly bounded as subsets of the linear system $F_{X}\left(F_{L}\right)$ and let $F^{-}$be the set of all members of $L^{*}\left(X^{*}\right)$ which carry every member of $B_{F}$ into a bounded set of real numbers. We state the important facts about this correspondence in a lemma.

Lemma. Let $X, L, \mathcal{F}_{1}, \mathcal{F}_{2}$ and $\left(^{-}\right)$be as in the immediately preceding paragraph and let $F$ and $G$ be members of $\mathcal{F}_{1}\left(\mathcal{F}_{2}\right)$ such that $F \subseteq G$. Then $F^{-} \supseteq G^{-}$, $F^{-} \subseteq F, F^{-}=F^{-}$, and if $F$ is a norm set so is $F^{-}$.

Proof. The first two statements are immediate consequences of the definitions. The third follows from the first two as follows $\left.{ }^{34}\right)$. Since $F-\subseteq F$, $F^{-}=\left(F^{-}\right)-\supseteq F^{-}$and since $\left(F^{-}\right)^{-} \subseteq F^{-}, F^{-} \subseteq \subseteq F^{-}$. Thus $F^{-}=F^{-}$. The last statement follows at once from the fact that the uniformly bounded sub-

(as) We regard $X \subseteq \tilde{X} \subseteq L^{*}$.

(अ) Cf. footnote 8 . 
sets of the conjugate of the linear system of a normed linear space are the norm bounded ones.

If we say that a member $F$ of $\mathcal{F}_{1}\left(\mathscr{F}_{2}\right)$ such that $F^{-}=F$ contains $L(X)$ regularly we may state our quasi-analogue of Theorem VII-6 as follows.

Theorem VII-11. Let $X$ be a linear space and let $L$ be a total relatively bounded subspace of $\left(X^{*}\right)_{X}$. Let $L_{1} \supseteq L_{2} \supseteq L_{3} \ldots$ be a descending sequence of norm sets each of which contains $L$ regularly. Then $M=\prod L_{n}$ is a norm set $\left.{ }^{35}\right)$ if and only if there exists $n_{0}=1,2, \cdots$ such that $L_{n_{0}}=L_{n_{0}+1}=\cdots$.

Proof. The truth of this theorem is an immediate consequence of the following lemma for which we shall have further use below.

Lemma. Let $L_{1}, L_{2}, \cdots, M$ and $L$ be as in the statement of Theorem VII-11. Then if $N$ is any norm set containing $M$ there exists a positive integer $n_{0}$ such that $N$ contains $L_{n_{0}}$.

Proof. Let $L_{0}=L_{1}^{-}+L_{2}^{-} \dot{+} \cdots$. Since each $L_{n}^{-}$is a norm set it follows from Theorem V-13 that $L_{0}$ is boundedly closed. $M$, being an intersection of boundedly closed subspaces, is also boundedly closed. Thus we may form $M^{-}$and $L_{0}^{-}$. For each $n=1,2, \cdots, L_{n}^{-} \subseteq L_{0}$ and $M \subseteq L_{n}$. Hence $L_{0}^{-} \subseteq L_{n}^{--}=L_{n}$ and $L_{n}^{-} \subseteq M^{-}$so that $L_{0}^{-} \subseteq \prod L_{n}=M$ and $L_{0}=\sum_{n=1}^{\infty} L_{n}^{-} \subseteq M^{-}$. Applying (-) to $L_{0}^{-} \subseteq M$ and combining with $L_{0} \subseteq M^{-}$we obtain $L_{0}^{--} \supseteq M^{-} \supseteq L_{0}$. But since $L_{0}^{--} \subseteq L_{0}$ we conclude that $L_{0}^{-}=L_{0}=M^{-}$. In other words, $\left(\prod_{n=1}^{\infty} L_{n}\right)^{-}=\sum_{n=1}^{\infty} L_{n}^{-}$. Now since $N \supseteq M, N^{-} \subseteq M^{-}=\sum_{n=1}^{\infty} L_{n}^{-}$. For each $n=1,2, \cdots$, let $N_{n}=L_{n}^{-} \cap N^{-}$. Then $N^{-}=N_{1} \dot{+} N_{2} \dot{+} \cdots$. Since $N^{-}$and each $N_{n}$ is a norm set it follows from Theorem VII-6 that for some $n_{0}=1,2, \cdots, N^{-}=N_{1}+N_{2} \dot{+} \cdots+N_{n_{0}}=N_{n_{0}}$. Hence $N^{-} \subseteq L_{n_{0}}^{-}$so that $N^{-} \supseteq L_{n_{0}}=L_{n_{0}}$. Since $N \supseteq N^{--}, N \supseteq L_{n_{0}}$ and the proof of the lemma is complete.

We are forced to restrict ourselves to descending sequences in the statement of Theorem VII-11 because we do not know that the intersection of two subspaces of $\left(X^{*}\right)_{X}$ each of which contains $L$ regularly need also contain $L$ regularly.

As the reader may readily verify using the theorems of this chapter, if $L$ is any relatively bounded, boundedly closed total subspace of an $\left(X^{*}\right)_{X}$ and $L$ is not a norm set, then there exists a sequence $L_{1}, L_{2}, \cdots$ of norm sets each of which contains its successor properly and $L$ regularly. Thus Theorem VII-11 is not vacuous and gives us a second method of constructing countable intersections of norm sets which are not pseudo-norm sets

The following theorem, whose proof we leave to the reader, throws some light on the nature of regular inclusion.

TieOREM VII-12. Let $X$ be a linear space and let $L$ be a total relatively

${ }^{(35)}$ Since $M$ is total it is a norm set if and only if it is a pseudo-norm set. 
bounded subspace of $\left(X^{*}\right)_{X}$. Then a norm set containing $L$ contains $L$ regularly if and only if there is a subset $K$ of $L$ such that $N$ is the norm set of the norm $\|$. defined as follows. $\|x\|=1$.u.b. $l \in K|l(x)|$ for all $x$ in $X$.

We now make use of the lemma associated with Theorem VII-11 to characterize countable intersections of norm sets in terms of the concepts of the fourth and fifth chapters.

THEOREM VII-13. Let $X$ be a linear space and let $L$ be a subspace of $\left(X^{*}\right)_{X}$. Then $L$ is the intersection of countably many norm sets if and only if it is boundedly closed, relatively bounded and has the second countability property.

Proof. Since we already know that an intersection of norm sets is boundedly closed and relatively bounded we may suppose at the outset that $L$ has these two properties. Since $L$ is relatively bounded it follows that every $L$ bounded subset of $X$ is $N$ bounded for some pseudo-norm set $N$ which contains $L$. In fact if $A$ is $L$ bounded, then $A \subseteq B$ where $\widetilde{C} o(B)=B$ and $B \dot{+}=X$, and by the procedure indicated in proving Theorem V-13 we may construct a pseudo-norm \|\| such that $\|x\| \leqq 1$ for all $x$ in $B$ and $\|x\| \geqq 1$ for all $x$ not in $B$. Let $N$ be the pseudo-norm set of \|\| . It is obvious that $L \subseteq N$ and that $A$ is $N$ bounded. Thus a subset of $X$ is $L$ bounded if and only if it is $N$ bounded for some pseudo-norm set $N$ containing $L$. It follows at once that $L$ has the second countability property if and only if there exists a countable family of pseudo-norm sets $L_{1} \supseteq L_{2} \supseteq L_{3} \supseteq \ldots$ all containing $L$ such that any pseudo-norm set containing $L$ contains some $L_{n}$. Now suppose that $L$ has the second countability property and let $L_{1}, L_{2}, \cdots$ be such a "basis of pseudo-norm sets." Let $l$ be any member of $X^{*}-L$. Since $L$ is relatively bounded and boundedly closed there exists a norm set $N$ which contains $L$ and not $l$. Let $n=1,2, \cdots$ be such that $L_{n} \subseteq N$. Then $L_{n}$ contains $L$ and not $l$. In other words $L=\prod_{n=1}^{\infty} L_{n}$. Since each $L_{n}$ is the intersection of two norm sets, $L$ is the intersection of countably many norm sets. Conversely suppose that $L$ is the intersection of countably many norm sets $L_{1}, L_{2}, \cdots$. Because of Theorem VII-7 we may suppose that $L_{1} \supseteq L_{2} \supseteq L_{3} \supseteq \cdots$. Thus if $L$ is total we may apply the lemma associated with Theorem VII-11 to the sequence $L_{1}^{--}, L_{2}^{--}, \cdots$ and conclude that any norm set containing $L$ contains some $L_{n}^{-}$and hence that $L$ has the second countability property. Suppose $L$ is not total. For each $n=1,2, \ldots$ let $M_{n}$ be the set of all $l$ in $L_{n}$ such that $l(x)=0$ for all $x$ in $L^{\prime}$. Then $L=\prod_{n=1}^{\infty} M_{n}$ and every $l$ in every $M_{n}$ vanishes throughout $L^{\prime}$. Thus when we pass to the quotient systems $X_{L} / L^{\prime}$ and $X_{M_{n}} / L^{\prime}$ we find that the corresponding subspaces of $\left(X / L^{\prime}\right)^{*}, \mathcal{L}$ and $\mathcal{X}_{n}$, are such that $\mathcal{L}=\prod_{n=1}^{\infty} \mathcal{X}_{n}$ and that the $\mathcal{X}_{n}$ are norm sets. Since $X_{L}$ is isomorphic to the direct union of $X_{L} / L^{\prime}$ and a linear system with only the zero functional distinguished and since $X_{L} / L^{\prime}$, being regular, has the second countability property by the argument just presented, it follows that $X_{L}$ does also. This completes the proof of the theorem. 
CoRollary. If $L_{1}, L_{2}, \cdots$ are subspaces of $\left(X^{*}\right)_{X}$ having the second countability property and if each $L_{n}$ is relatively bounded and boundedly closed then $\prod_{n=1}^{\infty} L_{n}$ also has the second countability property.

It follows from Theorem VII-6 that if $X$ is a linear space and $L$ and $M$ are subspaces of $\left(X^{*}\right)_{X}$ such that $L$ is a pseudo-norm set, $M$ is $\aleph_{0}$-dimensional, and $M \cap N=0$, then $L+M$ is not a pseudo-norm set. We conclude this section by applying Theorem VII-10 to obtain a generalization of this result.

Theorem VII-14. Let $X$ be a linear space and let $L$ and $M$ be subspaces of $\left(X^{*}\right)_{X}$ such that $L \cap M=0$. Then if $L$ is boundedly closed and $M$ is $\aleph_{0}$-dimensional; $L+M$ is not a pseudo-norm set.

Proof. Suppose that $L+M$ is a pseudo-norm set. Then $L$ is relatively bounded and, since boundedly closed by hypothesis, is an intersection of norm sets. Let $M=M_{1} \dot{+} M_{2} \dot{+} \cdots$ where each $M_{n}$ is finite-dimensional. Now since $L$ is an intersection of norm sets and since the intersection of a finite number of norm sets is a pseudo-norm set it follows that for each $n=1,2, \ldots$ there is a pseudo-norm set $L_{n}$ such that $L_{n} \cap M_{n}=0$ and $L \subseteq L_{n}$. Let $N_{n}=L_{n} \cap(L+M)$. Since the deficiency of $N_{n}$ in $L \dot{+} M$ is at most $\boldsymbol{\aleph}_{0}$ it follows from Theorem VII-6 that it is finite. Furthermore $\prod_{n=1}^{\infty} N_{n}=(L \dot{+} M)$ $\cap \prod_{n=1}^{\infty} L_{n}=L$ since $\prod_{n=1}^{\infty} L_{n} \cap M=0$ and $\prod_{n-1}^{\infty} L_{n} \supseteq L$. Thus $L$ is an intersection of pseudo-norm sets each having finite deficiency in the pseudo-norm set $L+M$. Hence by Theorem VII-10, $L$ is a pseudo-norm set. But then by Theorem VII- $6, L+M$ is not a pseudo-norm set and we have a contradiction.

Corollary. If $L$ is a pseudo-norm set and $L_{1}$ has deficiency $\aleph_{0}$ in $L$ then $L_{1}$ is simple and has deficiency $\boldsymbol{\aleph}_{0}$ in its bounded closure.

4. Some examples. We apply here the results of this and earlier chapters to obtain the examples promised in the remarks following Theorem V-1.

(1) Simple linear systems. Let $\dot{X}$ be any linear space and let $L$ be any pseudo-norm set in $\left(X^{*}\right)_{X}$. Then $X_{L}$ is a simple linear system.

(2) Relatively bounded linear systems having the first countability property and not the second. Let $X$ be any infinite-dimensional linear space and let $N$ be any pseudo-norm set in $\left(X^{*}\right)_{X}$. Let $M$ be any infinite-dimensional pseudonorm set such that $N \cap M=0$. That $M$ exists follows from Theorem V-9. Finally let $l_{1}, l_{2}, \ldots$ be a sequence of linearly independent members of $M$ and let $L=N+l_{1}+l_{2} \dot{+} \cdots$. Then since $M$ and $N$ are relatively bounded, $X_{L}$ is relatively bounded and since $N+l_{1}+l_{2} \dot{+} \cdots+l_{n}$, being a pseudo-norm set, has the first countability property, it follows from Theorem V-11 that $X_{L}$ has the first countability property. If $X_{L}$ had the second countability property then it would be simple and the bounded closure of $L$ would be a pseudo-norm set. Now by Theorem $\mathrm{V}-13, L$ is boundedly closed and by Theorem VII-6, $L$ is not a pseudo-norm set. Thus $X_{L}$ does not have the second countability property. 
(3) Relatively bounded linear systems having the second countability property and not the first. Let $X$ be an infinite-dimensional linear space and let $L$ be a subspace of $\left(X^{*}\right)_{X}$ which is not a pseudo-norm set but which is the intersection of countably many norm sets. We have already observed that such $L$ 's exist ${ }^{(36)}$. It follows from Theorem VII-13 that $X_{L}$ is relatively bounded, boundedly closed, and has the second countability property. Since $L$ is boundedly closed and not a pseudo-norm set it cannot have both countability properties. Thus $X_{L}$ does not have the first countability property.

(4) Relatively bounded linear systems with neither countability property. Let $X_{L}$ and $Y_{M}$ be relatively bounded linear systems such that $X_{L}$ has the first countability property and not the second and $Y_{M}$ has the second and not the first. It follows from Theorem V-3 that $X_{L} \oplus Y_{M}$ is relatively bounded and has neither countability property.

(5) A non relatively bounded linear system having the second countability property. Let $X$ be an $\aleph_{0}$-dimensional linear space. Obviously $X_{X}{ }^{*}$ has the second countability property. That it is not relatively bounded follows from Theorem V-6.

(6) Almost relatively bounded non relatively bounded linear systems with neither countability property. Let $X_{L}$ and $Y_{M}$ be linear systems such that $X_{L}$ is relatively bounded and has neither countability property and $Y_{M}$ is almost relatively bounded but not relatively bounded (for example, the example of (5)). Then by Theorem V-3, $X_{L} \oplus Y_{M}$ has neither countability property and is not relatively bounded but is almost relatively bounded.

(7) Non almost relatively bounded linear systems having the first countability property. We proceed as in (2), only this time requiring that $X$ be at least $C$-dimensional and choosing $l_{1}, l_{2}, \ldots$ so that $l_{1} \dot{+} l_{2} \dot{+} \ldots$ is not relatively bounded. That this is possible follows from Theorem V-7. As before, $X_{L}$ has the first countability property and not the second and now is not relatively bounded. Since it has the first countability property it follows from Theorem V-1 that it is not even almost relatively bounded.

(8) Non almost relatively bounded linear systems with neither countability property. Let $X$ be any linear space whose dimension exceeds $\aleph_{0}$. That $X_{X}$. has neither countability property and is not almost relatively bounded follows at once from the obvious fact that every bounded subset of $X_{X^{*}}$ has a finite-dimensional linear span.

\section{SOME UNSOLVED PROBLEMS}

We conclude with a list of a few of the questions and topics for further investigation suggested by the material developed above.

1. Is there an example of a regular infinite-dimensional linear system which is not stable?

${ }^{\left({ }^{36}\right)}$ See the corollary to Theorem V-14 and the remarks following Theorem VII-11. 
2. Are there any incomplete normed linear spaces in whose lattices of closed subspaces modularity and $d$-modularity are equivalent?

3 . Is the convergence in the linear system $X_{L}$ related in the general case to any of the topologies in $X$ with respect to which $X$ is a topological linear space in the sense of Kolmogoroff [12]?

4. If $X$ is a linear space and $L$ and $M$ are boundedly closed subspaces of $\left(X^{*}\right)_{X}$, when is $L+M$ boundedly closed?

(a) Is it always boundedly closed?

(b) Is it boundedly closed if $L$ and $M$ have the first countability property?

(c) Is it boundedly closed if one of $L$ and $M$ is $\boldsymbol{\aleph}_{0}$-dimensional?

(d) Is it boundedly closed if one of $L$ and $M$ is a pseudo-norm set?

5. Study completely boundedly closed linear systems.

6. Are there any relatively bounded linear systems which are boundedly closed but not completely boundedly closed?

7. Is the first countability property preserved under finite intersections?

8. Is the second countability property preserved under countable intersections when bounded closure and relative boundedness are not assumed?

9. Is every subspace of an $\left(X^{*}\right)_{X}$ with the first countability property a linear union of countably many pseudo-norm sets?

10. Is the reach of a linear system necessarily boundedly closed?

11. Is the operation of taking the reach idempotent?

12. Is every almost complete normed linear space a space of the second category?

13. Study the concept of regular inclusion more closely. In particular if $M_{1}$ and $M_{2}$ contain $L$ regularly does $M_{1} \cap M_{2}$ contain $L$ regularly?

14. Relate linear systems to vector lattices. In particular what linear systems are of the form $X_{L}$ where $X$ is a vector lattice and $L$ is the set of bounded linear functionals on $L$ ?

\section{BIBLIOGRAPHY}

1. B. H. Arnold, Rings of operators on vector spaces, Ann. of Math. vol. 45 (1944) pp. 24-49.

2. S. Banach, Theorie des operations lineaires, Warsaw, 1932.

3. G. Birkhoff, Lattice theory, Amer. Math. Soc. Colloquium Publications, vol. 25, New York, 1940.

4. R. P. Boas and J. W. Tukey, A note on linear functionals, Bull. Amer. Math. Soc. vol. 44 (1938) pp. 523-528.

5. M. Eidelheit, On isomorphisms of rings of linear operators, Studia Mathematica vol. 9 (1940) pp. 97-105.

6. G. Fichtenholz, Sur les fonctionelles linéaires continues au sens generalisê, Rec. Math. (Mat. Sbornik) N.S. vol. 4 (1938) pp. 193-213.

7. G. Hamel, Eine Basis aller Zahlen und die unstetige Lösungen der Funktional Gleichung $f(x+y)=f(x)+f(y)$, Math. Ann. vol. 60 (1905) pp. 459-462.

8. F. Hausdorff, Zur Theorie der linearen metrischen Räume, J. Reine Angew. Math. vol. 167 (1932) pp. 294-311.

9. D. H. Hyers, A note on linear topological spaces, Bull. Amer. Math. Soc. vol. 44 (1938) pp. 76-80. 
10. O. D. Kellogg, Foundations of potential theory, Berlin, 1929.

11. H. Kober, A theorem on Banach spaces, Compositio Math. vol. 7 (1939) pp. 135-140.

12. A. Kolmogoroff, Zur Normierbarkeit eines allgemeinen topologischen linearen Raumes, Studia Mathematica vol. 5 (1935) pp. 29-33.

13. M. Krein and V. Smulian, On regularly convex sets in the space conjugate to a Banach space, Ann. of Math. vol. 41 (1940) pp. 556-583.

14. E. R. Lorch, On a calculus of operators in reflexive vector spaces, Trans. Amer. Math. Soc. vol. 45 (1939) pp. 217-234.

15. H. Löwig, Über die Dimension linearer Räume, Studia Mathematica vol. 5 (1934) pp. 18-23.

16. G. W. Mackey, Isomorphisms of normed linear spaces, Ann. of Math. vol. 43 (1942) pp. 244-260.

17. - On infinite dimensional linear spaces, Proc. Nat. Acad. Sci. U.S.A. vol. 29 (1943) pp. 216-221.

18. - On convex topological linear spaces, Proc. Nat. Acad. Sci. U.S.A. vol. 29 (1943) pp. 315-319.

19. - Equivalence of a problem in measure theory to a problem in the theory of vector lattices, Bull. Amer. Math. Soc. vol. 50 (1944) pp. 719-722.

20. S. Mazur, Über konvexe Mengen in linearen normierten Räumen, Studia Mathematica vol. 4 (1933) pp. 70-84.

21. J. von Neumann, On complete topological spaces, Trans. Amer. Math. Soc. vol. 37 (1935) pp. 1-20.

22. G. Sirvint, Espace de fonctionelles linéaires, C. R. (Doklady) Acad. Sci. URSS. vol. 26 (1940) pp. 123-126.

23. M. H. Stone, Linear transformations in Hilbert space and their applications to analysis, Amer. Math. Soc. Colloquium Publications, vol. 15, New York, 1932.

24. J. W. Tukey, Convergence and uniformity in topology, Princeton, 1940.

25. S. Ulam, Zur Masstheorie in der allgemeinen Mengenlehre, Fund. Math. vol. 16 (1930) pp. $140-150$.

26. J. V. Wehausen, Transformations in linear topological spaces, Duke Math. J. vol. 4 (1938) pp. 157-169.

27. A. Weil, L'integration dans les groupes topologiques et ses applications, Paris, 1938.

28. L. R. Wilcox, Modularity in the theory of lattices, Ann. of Math. vol. 40 (1939) pp. 490505.

\section{HaRVARD UNIVERSITY,}

Cambridge, Mass. 